\title{
CONVERGENCE ANALYSIS OF MESHFREE APPROXIMATION SCHEMES*
}

\author{
A. BOMPADRE ${ }^{\dagger}$, B. SCHMIDT SCAND M. ORTIZ $^{\ddagger}$
}

\begin{abstract}
This work is concerned with the formulation of a general framework for the analysis of meshfree approximation schemes and with the convergence analysis of the local maximum-entropy (LME) scheme as a particular example. We provide conditions for the convergence in Sobolev spaces of schemes that are $n$-consistent in the sense of exactly reproducing polynomials of degree less than or equal to $n \geq 1$ and whose basis functions are of rapid decay. The convergence of the LME in $W_{\text {loc }}^{1, p}(\Omega)$ follows as a direct application of the general theory. The analysis shows that the convergence order is linear in $h$, a measure of the density of the point set. The analysis also shows how to parameterize the LME scheme for optimal convergence. Because of the convex approximation property of LME, its behavior near the boundary is singular and requires additional analysis. For the particular case of polyhedral domains we show that, away from a small singular part of the boundary, any Sobolev function can be approximated by means of the LME scheme. With the aid of a capacity argument, we further obtain approximation results with truncated LME basis functions in $H^{1}(\Omega)$ and for spatial dimension $d>2$.
\end{abstract}

Key words. meshfree interpolation, convergence analysis, error bounds

AMS subject classifications. $65 \mathrm{~N} 12,65 \mathrm{~N} 15,90 \mathrm{C} 25$

DOI. $10.1137 / 110828745$

1. Introduction. Meshfree approximation schemes (see, e.g., [14] for a review) are advantageous in a number of areas of application, e.g., those involving Lagrangian descriptions of unconstrained flows (see, [17] for a representative example), where methods based on triangulation, such as the finite element method, inevitably suffer from problems of mesh entanglement. The present work is concerned with the formulation of a general framework for the analysis of meshfree approximation schemes (see, e.g., [18] for representative past work) and with its application to the local maximum-entropy (LME) scheme as an example. By way of conceptual backdrop, we may specifically envision time-independent problems for which the solutions of interest follow as the minimizers of a functional $F: X \rightarrow \overline{\mathbb{R}}$, where $X$ is a topological vector space. General conditions for the existence of solutions are provided by Tonelli's theorem (e.g., [11]). In this framework, an approximation scheme is a sequence $X_{k}$ of subspaces of $X$, typically of finite dimension, defining a corresponding sequence of Galerkin reductions of $F$ :

$$
F_{k}(u)= \begin{cases}F(u) & \text { if } u \in X_{k} \\ +\infty & \text { otherwise }\end{cases}
$$

*Received by the editors March 28, 2011; accepted for publication (in revised form) April 9, 2012; published electronically May 29, 2012. This work was supported by the Department of Energy National Nuclear Security Administration under award DE-FC52-08NA28613 through Caltech's ASC/PSAAP Center for the Predictive Modeling and Simulation of High Energy Density Dynamic Response of Materials.

http://www.siam.org/journals/sinum/50-3/82874.html

${ }^{\dagger}$ Graduate Aerospace Laboratories, California Institute of Technology, Pasadena, CA 91125 (abompadr@caltech.edu).

${ }^{\ddagger}$ Institut für Mathematik, Universität Augsburg, Universitätsstr. 14, 86157 Augsburg, Germany (bernd.schmidt@math.uni-augsburg.de).

$\S$ Corresponding author. Graduate Aerospace Laboratories, California Institute of Technology, Pasadena, CA 91125 (ortiz@aero.caltech.edu). 
An approximation scheme is then said to be convergent if it has the following density property: For every $u \in X$, there exists a sequence $u_{k} \in X_{k}$ such that $\lim _{k \rightarrow \infty} u_{k}=u$. The connection between density of the approximation scheme and convergence is provided by the following proposition [8].

Proposition 1.1. Let $X$ be endowed with two metrizable topologies $S$ and $T$, with $T$ finer than $S$. Let $F: X \rightarrow \overline{\mathbb{R}}$ be coercive in $(X, S)$ and continuous in $(X, T)$. Let $X_{k}$ be a dense sequence of sets in $(X, T)$ and let $F_{k}$ be the corresponding sequence of Galerkin reductions of $F$. Then the sequence $F_{k} \Gamma$-converges to the lower semicontinuous envelope of $F$ and is equicoercive in $(X, S)$.

We recall that $\Gamma$-convergence is a powerful notion of variational convergence of functionals that, in particular, implies convergence of minimizers. Thus, if the sequence $F_{k}$ is equicoercive, then the minimizers of $F$ are accumulation points of minimizers of $F_{k}$; i.e., if $F_{k}\left(u_{k}\right)=\inf F_{k}$, then the sequence $u_{k}$ has a subsequence that converges to a minimizer of $F$. We also recall that the topology $T$ is finer than $S$; i.e., any converging sequence for $T$ converges for $S$. In applications, $T$ is typically a metric or normed topology and $S$ the corresponding weak topology.

It thus follows that, within the general framework envisioned here, the analysis of convergence of approximation schemes reduces to ascertaining the density property. Toward this end, in section 3 we begin by analyzing meshfree approximation schemes that are $n$-consistent in the sense of exactly reproducing polynomials of degree less than or equal to $n \geq 1$ and whose basis functions are of rapid decay. Specifically, for schemes subordinate to point sets possessing a certain geometrical regularity property that we term $h$-density, we prove a uniform error bound for consistent and rapidly decaying approximation schemes. In addition, we show that the sets of functions spanned by consistent and rapidly decaying approximation schemes are dense in Sobolev spaces.

In sections 4 and 5 , we apply the general results of section 3 to the LME approximation scheme of Arroyo and Ortiz [2] (see also [3, 30, 10, 13]). The LME scheme has been extensively assessed numerically over a broad range of test problems $[2,20,17]$, but a rigorous convergence analysis has been heretofore unavailable. The general theory of section 3 readily establishes the density of the LME approximation spaces $X_{k}$ in $W_{\text {loc }}^{1, p}(\Omega)$; see section 4 . In particular, the analysis shows that the convergence order is linear in $h$, a measure of the density of the point set. These convergence rates and the corresponding error bounds are in agreement with the numerical results reported in [2] and are comparable to those of the first-order finite element method (see, e.g., [6]). Conveniently, the analysis also shows how to choose the LME temperature parameter so as to obtain optimal convergence. This optimal choice is in agreement with that determined in $[2,17]$ by means of numerical testing.

The LME scheme is a convex approximation scheme in which the basis functions are constrained to take nonnegative values. By virtue of this restriction, the LME scheme is defined for convex domains only. Consequently, its behavior near the boundary is somewhat singular and requires careful additional analysis. In section 5 , for the particular case of polyhedral domains, we show that, away from a small singular part of the boundary, any Sobolev function can indeed be approximated by means of the LME scheme. Then, with the aid of a capacity argument, we obtain approximation results with truncated LME basis functions in $H^{1}(\Omega)$ and for spatial dimension $d>2$.

2. Prolegomena. The open $d$-ball $B(x, \delta)$ of radius $\delta$ centered at $x$ is the set $\left\{y \in \mathbb{R}^{d}:|y-x|<\delta\right\}$. The closed $d$-ball $\bar{B}(x, \delta)$ of radius $\delta$ centered at $x$ is the set $\left\{y \in \mathbb{R}^{d}:|y-x| \leq \delta\right\}$. Given a set $A \subset \mathbb{R}^{d}$, we denote by $\bar{A}$ its closure and by $\partial A$ its 
boundary. By a domain we shall specifically understand an open and bounded subset of $\mathbb{R}^{d}$. Given a point set $P \in\left(\mathbb{R}^{d}\right)^{N}$, we denote by $\overline{c o n v}(P)$ its closed convex hull [22] and by $\operatorname{conv}(P)$ the interior of $\overline{c o n v}(P)$. We recall that a $d$-simplex $T \subset \mathbb{R}^{d}$ is the convex hull of $d+1$ affinely independent points [22]. Given a bounded set $A \subset \mathbb{R}^{d}$, its size $h_{A}$ is the diameter of the smallest ball containing $A$.

The following definitions formalize the notion of a point set $P \subset \Omega$ that approximates a domain $\Omega$ uniformly.

Definition 2.1 ( $h$-covering). We say that a point set $P \subset \mathbb{R}^{d}$ is an $h$-covering of a set $A \subset \mathbb{R}^{d}, h>0$, if for every $x \in A$ there exists a d-simplex $T_{x}$ of size $h_{T_{x}}<h$ and with vertices in $P$ such that $x \in T_{x}$.

Definition 2.2 ( $h$-density). We say that a point set $P \subset \mathbb{R}^{d}$ has $h$-density bounded by $\tau>0$ if for every $x \in \mathbb{R}^{d}, \#(P \cap \bar{B}(x, h)) \leq \tau$.

For a point set $P \subset \Omega$ with $h$-density bounded by $\tau$, the following proposition bounds its number of points in rings of $\mathbb{R}^{d}$.

Proposition 2.3. Assume that $P \subset \Omega$ has h-density bounded by $\tau$ for some $h$, $\tau>0$. Then there is a constant $c>0$ that depends on $\tau$ and $d$ such that

$$
\#(P \cap(\bar{B}(x, t h) \backslash B(x,(t-1) h))) \leq c t^{d-1}
$$

for all $x \in \Omega$ and integers $t \geq 1$.

3. Convergence analysis of general meshfree approximation schemes. In this section we analyze meshfree approximation schemes that are $n$-consistent and whose basis functions (also known as shape functions in the finite element literature) are of rapid decay. Specifically, we prove a uniform error bound for consistent and rapidly decaying approximation schemes. In addition, we show that the set of functions spanned by consistent and rapidly decaying approximation schemes are dense in Sobolev spaces.

Let $\Omega \subset \mathbb{R}^{d}$ be a domain. By an approximation scheme $\{I, W, P\}$, we mean a collection $W=\left\{w_{a}, a \in I\right\}$ of basis functions and a point set $P$, both indexed by $I$. Given an approximation scheme $\{I, W, P\}$, we approximate functions $u: \Omega \rightarrow \mathbb{R}$ by functions in the span $X$ of $W$ of the form

$$
u_{I}(x)=\sum_{a \in I} u\left(x_{a}\right) w_{a}(x)
$$

provided that this operation is well defined. More generally, we shall consider sequences of approximation schemes $\left\{I_{k}, W_{k}, P_{k}\right\}$ and let

$$
u_{k}(x)=\sum_{a \in I_{k}} u\left(x_{a}\right) w_{a}(x)
$$

be the corresponding sequence of approximations to $u$ in the sequence $X_{k}$ of finitedimensional spaces of functions spanned by $W_{k}$. We note that, for simplicity, we assume that all functions are defined over a common domain $\Omega$. Depending on the approximation scheme, this assumption may implicitly restrict the type of domains that may be considered, e.g., polyhedral domains. The aim then is to ascertain conditions on the approximation scheme under which $u_{k} \rightarrow u$ in an appropriate Sobolev space $W^{m, p}(\Omega)$.

We recall the following definition of consistency of approximation schemes [26].

DeFINITION 3.1 (consistency). We say that an approximation scheme $\{I, W, P\}$ is consistent of order $n \geq 0$ or $n$-consistent, relative to a point set $P$ if it exactly interpolates polynomials of degree less than or equal to $n$ within $\Omega$; i.e., if 


$$
x^{\alpha}=\sum_{a \in I} x_{a}^{\alpha} w_{a}(x)
$$

for all multi-indices $\alpha$ of degree $|\alpha| \leq n$.

We recall that the Taylor approximation of order $r$ of a function $u \in C^{r+1}(\Omega)$ at $y \in \Omega$ is

$$
T_{r}(u)(x, y)=\sum_{|\alpha| \leq r} \frac{1}{\alpha !} D^{\alpha} u(x)(y-x)^{\alpha}
$$

and its remainder is

$$
R_{r+1}(x, y)=u(y)-T_{r}(u)(x, y)=\sum_{|\alpha|=r+1} \frac{1}{\alpha !} D^{\alpha} u(x+\theta(y-x))(y-x)^{\alpha}
$$

for some $\theta \in(0,1)$.

Functions in the span of a consistent basis-function set satisfy the following multipoint Taylor formula (see [6, 7]). Its proof follows that of Theorem 1 of [7].

Proposition 3.2 (multipoint Taylor formula). Let $W$ be a $C^{r}(\Omega)$ basis-function set $n$ th-order consistent relative to a point set $P$ in $\Omega, u \in C^{\ell+1}(\operatorname{conv}(\Omega))$ and $m=$ $\min \{n, \ell\}$. Then,

$$
D^{\alpha} u_{I}(x)=D^{\alpha} u(x)+\sum_{a \in I} R_{m+1}\left(x_{a}, x\right) D^{\alpha} w_{a}(x)
$$

for all $|\alpha| \leq \min \{m, r\}$ and $x \in \Omega$.

We recall that a function $f \in C^{\infty}\left(\mathbb{R}^{d}\right)$ is said to be rapidly decreasing if [23]

$$
\sup _{|\alpha| \leq N} \sup _{x \in \mathbb{R}^{d}}\left(1+|x|^{2}\right)^{N}\left|\left(D^{\alpha} f\right)(x)\right|<\infty
$$

for all $N=0,1,2, \ldots$, where $|x|^{2}=\sum x_{i}^{2}$.

The next definition formalizes a polynomial decay condition of the basis functions and their derivatives.

DEFINITION 3.3 (approximation scheme with polynomial decay). We say that an approximation scheme $\{I, W, P\}$ has a polynomial decay of order $(r, s)$ for constants $c>0$ and $h>0$ if the basis $W$ is in $C^{r}(\Omega)$ and

$$
\sup _{|\alpha| \leq r} \sup _{x \in \Omega} \sup _{a \in I}\left(1+\left|\frac{x-x_{a}}{h}\right|^{2}\right)^{s} h^{|\alpha|}\left|D^{\alpha} w_{a}(x)\right|<c .
$$

A sequence of approximation schemes $\left\{I_{k}, W_{k}, P_{k}\right\}$ has a uniform polynomial decay of order $(r, s)$ if there exist a constant $c>0$ and a sequence $h_{k} \rightarrow 0$ such that, for each $k,\left\{I_{k}, W_{k}, P_{k}\right\}$ has a polynomial decay of order $(r, s)$ for constants $c$ and $h_{k}$.

We note that, if the basis functions are invariant under a linear transformation, then the left-hand side of (3.8) is also invariant under the same transformation change.

The next proposition establishes a key concentration property of approximation schemes with polynomial decay.

Proposition 3.4 (basis function concentration). Let $\{I, W, P\}$ be an approximation scheme. Suppose that there exists $\tau>0$ such that $P$ has $h$-density bounded by $\tau$. Suppose, in addition, that the approximation scheme has polynomial decay of order 
$(r, s)$ for constants $c>0$ and $h$, with $2 s>d$. Then, for every $\theta>0$, there exists a constant $c_{\theta}>0$ such that

$$
\sum_{x_{a} \in P \backslash \bar{B}\left(x, c_{\theta} h\right)}\left|w_{a}(x)\right| \leq \theta,
$$

everywhere in $\Omega$.

Proof. For every nonnegative integer $t \geq 1$, let $U_{t}(x)$ be the ring of node points $U_{t}(x)=\left\{x_{a} \in P:(t-1) h \leq\left|x_{a}-x\right|<t h\right\}$. Combining the bound on the number of points of $U_{t}(x)$ given by Proposition 2.3 with the assumption of polynomial decay of the approximation scheme, for any integer $c_{\theta} \geq 1$ we have

$$
\begin{aligned}
\sum_{x_{a} \in P \backslash \bar{B}\left(x, c_{\theta} h\right)}\left|w_{a}(x)\right| & \leq \sum_{t=c_{\theta}}^{\infty} \sum_{x_{a} \in U_{t}(x)}\left|w_{a}(x)\right| \leq \sum_{t=c_{\theta}}^{\infty} c^{\prime} t^{d-1} c\left((t-1)^{2}+1\right)^{-s} \\
& \leq \sum_{t=c_{\theta}}^{\infty} c^{\prime} t^{d-1} c t^{-2 s} \leq \sum_{t=c_{\theta}}^{\infty} c^{\prime} c t^{-1-(2 s-d)}
\end{aligned}
$$

Note that the series $\sum_{t=1}^{\infty} c^{\prime} c t^{-1-(2 s-d)}$ is finite. In particular, there exists a value $c_{\theta}<\infty$, depending on $d, \tau$, and $\theta$, such that $\sum_{t=c_{\theta}}^{\infty} c^{\prime} c t^{-1-(2 s-d)} \leq \theta$.

For an $n$-consistent approximation scheme with sufficiently high polynomial decay, the following theorem provides a uniform interpolation error bound.

THEOREM 3.5 (uniform interpolation error bound). Let $\{I, W, P\}$ be an approximation scheme. Suppose that

(i) the approximation scheme is $n$-consistent, $n \geq 0$;

(ii) there exists $\tau>0$ such that $P$ has $h$-density bounded by $\tau$;

(iii) the approximation scheme has polynomial decay of order $(r, s)$ with $2 s>$ $d+m+1$, where $m=\min \{n, \ell\}$.

Let $u \in C^{\ell+1}(\overline{\operatorname{conv}}(\Omega))$. Then, there exists a constant $C<\infty$ such that

$$
\left|D^{\alpha} u_{I}(x)-D^{\alpha} u(x)\right| \leq C\left\|D^{m+1} u\right\|_{\infty} h^{m+1-|\alpha|}
$$

for every $|\alpha| \leq \min \{m, r\}$ and $x \in \Omega$.

Proof. By Proposition 3.2,

$$
\left|D^{\alpha} u_{I}(x)-D^{\alpha} u(x)\right| \leq \sum_{a \in I}\left|R_{m+1}\left(x_{a}, x\right)\right|\left|D^{\alpha} w_{a}(x)\right|
$$

for every multi-index $\alpha$ of degree less than or equal to $\min \{m, r\}$ and every $x \in \Omega$. Next, we proceed to bound the right-hand side of this inequality. For each nonnegative integer $t \geq 1$, let $U_{t}(x)$ be the ring of nodal points $U_{t}(x)=\left\{x_{a} \in P:(t-1) h \leq\right.$ $\left.\left|x_{a}-x\right|<t h\right\}$. Note that $P=\cup_{t=1}^{\infty} U_{t}(x)$. By Proposition 2.3, there exists a constant $c$ that depends on $\tau$ and $d$ such that, for any $t \geq 1$, the number of node points of $U_{t}(x)$ is at most $\# U_{t}(x) \leq c t^{d-1}$. In addition, from (3.5) we have

$$
\left|R_{m+1}\left(x_{a}, x\right)\right| \leq \frac{d^{m+1}}{(m+1) !}\left\|D^{m+1} u\right\|_{\infty}(t h)^{m+1} .
$$

By the assumption of polynomial decay there exists a constant $0<c^{\prime}<\infty$ such that

$$
\left|D^{\alpha} w_{a}(x)\right| \leq c^{\prime}\left(\left|\frac{x-x_{a}}{h}\right|^{2}+1\right)^{-s} h^{-|\alpha|} \leq c^{\prime}\left((t-1)^{2}+1\right)^{-s} h^{-|\alpha|} \leq 5 c^{\prime} t^{-2 s} h^{-|\alpha|}
$$


for every $x_{a} \in U_{t}(x)$. From the preceding bounds we have

$$
\begin{aligned}
\sum_{a \in I}\left|R_{m+1}\left(x_{a}, x\right)\right|\left|D^{\alpha} w_{a}(x)\right| & =\sum_{t=1}^{\infty} \sum_{x_{a} \in U_{t}(x)}\left|R_{m+1}\left(x_{a}, x\right)\right|\left|D^{\alpha} w_{a}(x)\right| \\
& \leq c^{\prime \prime}\left\|D^{m+1} u\right\|_{\infty} h^{m+1-|\alpha|} \sum_{t=1}^{\infty} t^{d+m-2 s}
\end{aligned}
$$

for a constant $c^{\prime \prime}$ that depends on $\tau, d$, and $s$. Since $d+m-2 s<-1$, it follows that $\sum_{t=1}^{\infty} t^{d+m-2 s}<\infty$. Thus,

$$
\left|D^{\alpha} u_{I}(x)-D^{\alpha} u(x)\right| \leq C\left\|D^{m+1} u\right\|_{\infty} h^{m+1-|\alpha|}
$$

for every $x \in \Omega$, where the constant $C$ depends on $\tau, d$, and $s$.

Similar error bounds are known to hold for $n$-consistent meshfree methods; see, e.g., $[18,4,19,24]$. These results are obtained assuming that the size of the supports of the basis functions goes to zero with the density of the node set and assuming a "finite overlap" restriction on the supports. In contrast, the polynomial decay condition of the basis functions of Theorem 3.5 can be seen as a weakening of these constraints.

The following corollaries to Theorem 3.5 show that a function in $W^{m, p}(\Omega)$ can be approximated by means of consistent approximation schemes of polynomial decay.

COROLlary 3.6. Under the assumptions of Theorem 3.5,

$$
\left\|u_{I}-u\right\|_{W^{j, p}(\Omega)} \leq C\left\|D^{m+1} u\right\|_{\infty} h^{1+m-j}
$$

for $1 \leq p<\infty, j=\min \{n, r, \ell\}$ and every $u \in C^{\ell+1}(\overline{\operatorname{conv}}(\Omega))$.

Proof. By Theorem 3.5, there exists a constant $0<C<\infty$ such that

$$
\left\|u_{I}-u\right\|_{C^{j}(\bar{\Omega})} \leq C\left\|D^{m+1} u\right\|_{\infty} h^{1+m-j}
$$

thus, the assertion follows from the continuous embedding $C^{j}(\bar{\Omega}) \hookrightarrow W^{j, p}(\Omega)$.

Convergence in $W^{j, p}(\Omega)$ finally follows from standard theory of approximation by continuous functions (see e.g., [1]). For completeness, we proceed to note a particular case of practical relevance. We recall that a domain $\Omega$ satisfies the segment condition if, for all $x$ in the boundary of $\Omega$, there exist a neighborhood $U_{x}$ and a direction $y_{x} \neq 0$ such that, for any point $z \in \bar{\Omega} \cap U_{x}$, the point $z+t y_{x}$ belongs to $\Omega$ for all $0<t<1$ (see [1, section 3.21]). Convex domains satisfy the segment condition without additional restrictions on their boundaries. We additionally recall that, if $\Omega$ satisfies the segment condition, then the functions of $C_{c}^{\infty}\left(\mathbb{R}^{d}\right)$ restricted to $\Omega$ are dense in $W^{j, p}(\Omega)$ for $1 \leq p<\infty$ (see [1, Theorem 3.22]).

Corollary 3.7. Let $\Omega$ be a domain satisfying the segment condition. Suppose that the assumptions of Theorem 3.5 hold uniformly for $h_{k} \rightarrow 0$. Let $1 \leq p<\infty$ and $j=\min \{n, r\}$. Then, for every $u \in W^{j, p}(\Omega)$, there exists a sequence $u_{k} \in X_{k}$ such that $u_{k} \rightarrow u$.

Proof. By the density of $C_{c}^{\infty}\left(\mathbb{R}^{d}\right)$ in $W^{j, p}(\Omega)$, there is a sequence of functions $v_{i} \in C_{c}^{\infty}\left(\mathbb{R}^{d}\right)$ whose restrictions to $\Omega$ converge to $u$ in $W^{j, p}(\Omega)$. The corollary then follows by approximating each $v_{i}$ by a sequence $u_{i_{k}} \in X_{k}$ and passing to a diagonal sequence. 
4. Application to the local maximum-entropy approximation scheme: Interior estimates. In this section, we specialize the results of section 3 to the LME approximation schemes. We begin with a brief review of the definition and some of the properties of the LME approximation scheme of Arroyo and Ortiz [2] (see also [3, 30] for a description of the method and $[27,28,29]$ for related work). We recall that a convex approximation scheme is a first-order consistent approximation scheme $\{I, W, P\}$ whose basis functions are nonnegative. Convex approximation schemes satisfy a weak Kronecker-delta property at the boundary (cf. [2]); i.e., the approximation on the boundary of the domain does not depend on the nodal data over the interior points. This property simplifies the enforcement of essential boundary conditions. As pointed out in [2], in a convex approximation scheme, the basis functions $w_{a}(x), a \in I$, are well defined if and only if $x \in \overline{c o n v}(P)$. Therefore, for such schemes to be feasible, the domain $\Omega$ must be a subset of $\overline{c o n v}(P)$.

The LME approximation scheme [2] is a convex approximation scheme that aims to satisfy two objectives simultaneously:

1. unbiased statistical inference based on the nodal data;

2. basis functions of least width.

Since for each point $x$ the basis functions of a convex approximation scheme are nonnegative and add up to 1, they can be thought of as the probability distribution of a random variable. The statistical inference of the basis functions is then measured by the entropy of the associated probability distribution as defined in information theory $[25,15,16]$. The entropy of a probability distribution $p$ over $I$ is

$$
H(p)=-\sum_{a \in I} p_{a} \log p_{a},
$$

where $0 \log 0=0$. The least biased probability distribution $p$ is that which maximizes the entropy. In addition, the width of a nonnegative function $w$ about a point $\xi$ is identified with the second moment

$$
U_{\xi}(w)=\int_{\Omega} w(x)|x-\xi|^{2} d x .
$$

Thus, the width $U_{\xi}(w)$ measures how concentrated $w$ is about $\xi$. According to this measure of width, the most local approximation scheme is that which minimizes the total width

$$
U(W)=\sum_{a \in I} U_{a}\left(w_{a}\right)=\int_{\Omega} \sum_{a \in I} w_{a}(x)\left|x-x_{a}\right|^{2} d x .
$$

The LME approximation schemes combine the functionals (4.1) and (4.3) into a single objective. More precisely, for a parameter $\beta>0$, the LME approximation scheme is the minimizer of the functional

$$
F_{\beta}(W)=\beta U(W)-H(W)
$$

under the restriction of first-order consistency. Because of the local nature of this functional, it can be minimized pointwise, leading to the local convex minimization problem

$$
\left.\begin{array}{l}
\min f_{\beta}(x, w(x))=\sum_{a \in I} w_{a}(x)\left|x-x_{a}\right|^{2}+\frac{1}{\beta} \sum_{a \in I} w_{a}(x) \log w_{a}(x) \\
\text { subject to: } w_{a}(x) \geq 0, a \in I, \quad \sum_{a \in I} w_{a}(x)=1, \quad \sum_{a \in I} w_{a}(x) x_{a}=x .
\end{array}\right\}
$$


In the limit of $\beta \rightarrow \infty$ the function $f_{\beta}$ reduces to the power function of Rajan [21], whose minimizers define the piecewise-affine basis functions supported by the Delaunay triangulations associated with $P$.

Next we collect alternative characterizations of the LME basis functions based on duality theory. Let $Z: \mathbb{R}^{d} \times \mathbb{R}^{d} \rightarrow \mathbb{R}$ be the partition function

$$
Z(x, \lambda)=\sum_{a \in I} e^{-\beta\left|x-x_{a}\right|^{2}+\left\langle\lambda(x), x-x_{a}\right\rangle}
$$

of the point set. For every point $x \in \overline{c o n v}(P)$, the LME problem has a unique solution $\left\{w_{a}^{*}(x): a \in I\right\}$. Moreover, for every point $x \in \operatorname{conv}(P)$, the optimal basis functions $w_{a}^{*}(x)$ at $x$ are of the form

$$
w_{a}^{*}(x)=\frac{e^{-\beta\left|x-x_{a}\right|^{2}+\left\langle\lambda^{*}(x), x-x_{a}\right\rangle}}{\sum_{b \in I} e^{-\beta\left|x-x_{b}\right|^{2}+\left\langle\lambda^{*}(x), x-x_{b}\right\rangle}},
$$

where the vector $\lambda^{*}(x) \in \mathbb{R}^{d}$ minimizes the function

$$
\log Z(x, \lambda)=\log \left(\sum_{a \in I} e^{-\beta\left|x-x_{a}\right|^{2}+\left\langle\lambda, x-x_{a}\right\rangle}\right) .
$$

At points $x$ belonging to the boundary of conv $P$, the basis functions take expressions similar to (4.6) that solely involve the node points on the minimal face of $\overline{c o n v}(P)$ that contains $x$. The gradient of $\log Z(x, \lambda)$ with respect to $\lambda$ is

$$
r(x, \lambda) \equiv \frac{\partial}{\partial \lambda} \log Z(x, \lambda)=\sum_{a \in I} w_{a}(x, \lambda)\left(x-x_{a}\right) .
$$

In addition, the Hessian of $\log Z(x, \lambda)$ with respect to $\lambda$ follows as

$$
J(x, \lambda) \equiv \frac{\partial^{2}}{\partial \lambda^{2}} \log Z(x, \lambda)=\sum_{a \in I} w_{a}(x, \lambda)\left(x-x_{a}\right) \otimes\left(x-x_{a}\right)-r(x, \lambda) \otimes r(x, \lambda) .
$$

Since $r\left(x, \lambda^{*}(x)\right)=0$,

$$
J^{*}(x) \equiv J\left(x, \lambda^{*}(x)\right)=\sum_{a \in I} w_{a}^{*}(x)\left(x-x_{a}\right) \otimes\left(x-x_{a}\right) .
$$

It can be shown that $J^{*}(x)$ is positive definite. In addition, the optimal basis functions $w_{a}^{*}: \operatorname{conv}(P) \rightarrow \mathbb{R}$ are $C^{\infty}$ and have gradient

$$
\nabla w_{a}^{*}(x)=-w_{a}^{*}(x)\left(J^{*}(x)\right)^{-1}\left(x-x_{a}\right) .
$$

We refer the reader to [2] for the proofs of the preceding results and identities.

The following lemma shows that, for a point set $P$ that is an $h$-covering of its closed convex hull $\overline{\operatorname{conv}}(P)$ and for every point $x \in \operatorname{conv}(P)$, for any vector $\lambda \neq 0$ there exists at least one node point $x_{\lambda}$ in $P$ that is close to $x$ such that $x-x_{\lambda}$ is closely aligned with $\lambda$.

Lemma 4.1. Let $P$ be a finite point set that is an h-covering of its convex hull $\overline{\operatorname{conv}}(P)$ for some $h>0$. Let $x$ be a point in $\operatorname{conv}(P)$. Let $\varepsilon>0$ be such that $\bar{B}(x, \varepsilon h) \subset \overline{\operatorname{conv}}(P)$. Let $\lambda \neq 0 \in \mathbb{R}^{d}$. Then, there exists a node point $x_{\lambda} \in P$ such that 
(i) $\varepsilon h \leq\left|x-x_{\lambda}\right| \leq(\varepsilon+1) h$;

(ii) $|\lambda| \varepsilon h \leq\left\langle\lambda, x-x_{\lambda}\right\rangle$.

Proof. Let $\tilde{x}$ be the point $\tilde{x}=x-\frac{\varepsilon h}{|\lambda|} \lambda$. Since the distance between $x$ and $\tilde{x}$ is $\varepsilon h, \tilde{x} \in \bar{B}(x, \varepsilon h) \subset \overline{c o n v}(P)$. In particular, since the point set $P$ is an $h$-covering of $\overline{c o n v}(P)$, there exists a $d$-simplex $T_{\tilde{x}}$ of size at most $h$, with vertices in $P$ that contains $\tilde{x}$. Let $H^{+} \subset \mathbb{R}^{d}$ be the halfspace $\left\{z \in \mathbb{R}^{d}:\langle\lambda, \tilde{x}-z\rangle \geq 0\right\}$. The point $\tilde{x}$ belongs to $H^{+}$. Moreover, since the $d$-simplex $T_{\tilde{x}}$ contains $\tilde{x}$, it follows that at least one extreme point of $T_{\tilde{x}}$ also belongs to $H^{+}$. Let $x_{\lambda}$ be that extreme point. Note that $x_{\lambda}$ is also a node point of $P$. We have the estimate

$$
\left|x-x_{\lambda}\right| \leq|x-\tilde{x}|+\left|\tilde{x}-x_{\lambda}\right| \leq \varepsilon h+h=(\varepsilon+1) h .
$$

In addition, we have

$$
\left|x-x_{\lambda}\right|^{2}=|x-\tilde{x}|^{2}+\left|\tilde{x}-x_{\lambda}\right|^{2}+2\left\langle x-\tilde{x}, \tilde{x}-x_{\lambda}\right\rangle .
$$

By the definition of $\tilde{x}$ and since $x_{\lambda}$ belongs to $H^{+}$, it follows that

$$
\left\langle x-\tilde{x}, \tilde{x}-x_{\lambda}\right\rangle=\frac{\varepsilon h}{|\lambda|}\left\langle\lambda, \tilde{x}-x_{\lambda}\right\rangle \geq 0 .
$$

From this inequality and (4.13) we obtain

$$
\left|x-x_{\lambda}\right|^{2} \geq|x-\tilde{x}|^{2}=(\varepsilon h)^{2}
$$

or $\left|x-x_{\lambda}\right| \geq \varepsilon h$. Finally, from the definition of $\tilde{x}$ we have

$$
\langle\lambda, x-\tilde{x}\rangle=|\lambda| \varepsilon h
$$

and

$$
\left\langle\lambda, x-x_{\lambda}\right\rangle=\langle\lambda, x-\tilde{x}\rangle+\left\langle\lambda, \tilde{x}-x_{\lambda}\right\rangle \geq|\lambda| \varepsilon h,
$$

where we have used that $x_{\lambda}$ belongs to $H^{+}$and, hence, $\left\langle\lambda, \tilde{x}-x_{\lambda}\right\rangle \geq 0$.

In view of (4.6), in order to verify that the LME basis functions have polynomial decay, we require a bound on the minimizer $\lambda^{*}(x) \in \mathbb{R}^{d}$ of the partition function $Z(x, \lambda)$, from (4.5) and (4.7). To this end, we begin with the following lemma.

LEMma 4.2. Let $P$ be a point set that is an $h$-covering of $\Omega$ with $h$-density bounded by $\tau$, for some $h, \tau>0$. Let $\beta=\frac{\gamma}{h^{2}}$ for some $\gamma>0$. Then, there exists a constant $c_{Z}$ that depends on $\gamma, \tau$, and $d$ such that

$$
Z(x, 0) \leq c_{Z}
$$

for every $x \in \operatorname{conv}(P)$.

Proof. For every nonnegative integer $t \geq 1$, let $U_{t}(x)$ be the subset of node points $U_{t}(x)=\left\{x_{a} \in P:(t-1) h \leq\left|x_{a}-x\right|<t h\right\}$. Then, by Proposition 2.3 we have

$$
\begin{aligned}
Z(x, 0) & =\sum_{a \in I} e^{-\beta\left|x-x_{a}\right|^{2}}=\sum_{t=1}^{\infty}\left(\sum_{x_{a} \in U_{t}(x)} e^{-\beta\left|x-x_{a}\right|^{2}}\right) \\
& \leq \sum_{t=1}^{\infty}\left(\# U_{t}(x) e^{-\beta(t-1)^{2} h^{2}}\right) \leq \sum_{t=1}^{\infty} c t^{d-1} e^{-\gamma(t-1)^{2}}=c_{Z} .
\end{aligned}
$$

Copyright $@$ by SIAM. Unauthorized reproduction of this article is prohibited. 
It is readily verified that the series on the right-hand side is absolutely convergent. Moreover, because this series is defined in terms of $\gamma, \tau$, and $d$, its limit $c_{Z}$ also depends on $\gamma, \tau$, and $d$ only.

By optimality, $\lambda^{*}(x)$ has the property that $Z\left(x, \lambda^{*}(x)\right) \leq Z(x, 0)$. This observation, combined with the upper bound on $Z(x, 0)$ of Lemma 4.2, suffices to estimate $\left|\lambda^{*}(x)\right|$.

LEMma 4.3. Let $P$ be a point set that is an $h$-covering of $\Omega$ with h-density bounded by $\tau$ for some $h, \tau>0$. Let $\beta=\frac{\gamma}{h^{2}}$ for some $\gamma>0$ and $\varepsilon>0$. Then, there exists a constant $c_{\lambda}>0$ that depends on $\gamma, \tau$, and d only such that

$$
\left|\lambda^{*}(x)\right| \leq \frac{c_{\lambda}}{\min \{\varepsilon, 1\} h}
$$

for every point $x$ such that $\bar{B}(x, \varepsilon h) \subset \overline{c o n v}(P)$.

Proof. We note that since $\log$ is an increasing function, $\lambda^{*}(x)$ also minimizes $Z(x, \lambda)$. Let $\varepsilon_{2}=\min \{\varepsilon, 1\}$. We proceed to find a constant $c_{\lambda}$ such that if $|\lambda| \geq \frac{c_{\lambda}}{\varepsilon_{2} h}$, then $Z(x, \lambda)>Z(x, 0)$. To this end, let $\lambda \neq 0$ be a fixed vector. Since $\bar{B}\left(x, \varepsilon_{2} h\right) \subset$ $\overline{\operatorname{conv}}(P)$ and $P$ is an $h$-covering of $\overline{c o n v}(P)$, by Lemma 4.1 there exists a point $x_{\lambda} \in P$ such that $\varepsilon_{2} h \leq\left|x-x_{\lambda}\right| \leq\left(\varepsilon_{2}+1\right) h$ and $\left\langle\lambda, x-x_{\lambda}\right\rangle \geq|\lambda| \varepsilon_{2} h$. Using these inequalities and noting that $\varepsilon_{2} \leq 1$, we further obtain

$$
Z(x, \lambda) \geq e^{-\beta\left|x-x_{\lambda}\right|^{2}+\left\langle\lambda, x-x_{\lambda}\right\rangle} \geq e^{-\beta\left(1+\varepsilon_{2}\right)^{2} h^{2}+\varepsilon_{2} h|\lambda|} \geq e^{-4 \gamma+\varepsilon_{2} h|\lambda|} .
$$

By Lemma 4.2 , there exists a constant $c_{Z}$ that depends on $\gamma, \tau$, and $d$ such that $Z(x, 0) \leq c_{Z}$. Combining this bound with (4.21), it follows that a sufficient condition for $\lambda$ not to be optimal is that $e^{-4 \gamma+\varepsilon_{2} h|\lambda|}>c_{Z}$ or, equivalently,

$$
|\lambda|>\frac{\ln c_{Z}+4 \gamma}{\min \{\varepsilon, 1\} h}=\frac{c_{\lambda}}{\min \{\varepsilon, 1\} h} .
$$

Therefore, (4.20) is a necessary condition for $\lambda^{*}(x)$ to be optimal.

We note that, for fixed $\varepsilon>0$ and for points $x$ at distance $\varepsilon$ or greater to the boundary of $\overline{c o n v}(P)$, the upper bound (4.20) is $\mathrm{O}\left(h^{-1}\right)$. By contrast, for points $x \in \overline{c o n v}(P)$ arbitrarily close to the boundary of $\overline{c o n v}(P)$, the right-hand side of (4.20) diverges. The following example shows that $\left|\lambda^{*}(x)\right|$ may indeed diverge near the boundary.

Example 1. Let $\Omega=[a, b] \subset \mathbb{R}, h=b-a$ and let $P=\{a, b\}$ be a point set of $\Omega$. Let $\beta=\frac{\gamma}{h^{2}}$ for some $\gamma>0$. The optimality condition for $\lambda^{*}(x)$ is

$\frac{\partial Z(x, \lambda)}{\partial \lambda}=e^{-\frac{\gamma}{h^{2}}(x-a)^{2}+\lambda^{*}(x)(x-a)}(x-a)+e^{-\frac{\gamma}{h^{2}}(x-a-h)^{2}+\lambda^{*}(x)(x-a-h)}(x-a-h)=0$.

For this condition we find

$$
\lambda^{*}(x)=\frac{\log (a+h-x)-\log (x-a)}{h}+\frac{\gamma}{h^{2}}(2 x-2 a-h) .
$$

For a fixed $0<\varepsilon<1$ and for points $x \in(a+\varepsilon h, a+h-\varepsilon h)$, we indeed have $\left|\lambda^{*}(x)\right|=\mathrm{O}\left(h^{-1}\right)$. However, $\lim _{x \rightarrow a^{+}} \lambda^{*}(x)=\infty$ and $\lim _{x \rightarrow b^{-}} \lambda^{*}(x)=-\infty$. We note that the LME basis functions for this case reduce to

$$
\begin{aligned}
& w_{a}^{*}(x)=\frac{a+h-x}{h}, \\
& w_{b}^{*}(x)=\frac{x-a}{h} .
\end{aligned}
$$

Copyright (c) by SIAM. Unauthorized reproduction of this article is prohibited. 
In particular, the basis functions and their derivatives are bounded in $\Omega$ even though the value of $\left|\lambda^{*}(x)\right|$ is unbounded at the boundary. From a computational perspective, this example suggests that computing the basis functions and their derivatives using (4.7) and (4.6) may be unstable near the boundary, even if the basis functions and their derivatives are themselves well-behaved. In section 5 we will examine the behavior of the basis functions near the boundary more thoroughly.

The following lemma supplies the requisite estimate of the partition function $Z$.

LEMma 4.4. Under the assumptions of Lemma 4.3, there exist constants $m_{Z}$, $M_{Z}>0$ that depend on $\gamma, \tau, \varepsilon$, and $d$ only and such that

$$
m_{Z} \leq Z\left(x, \lambda^{*}(x)\right) \leq M_{Z}
$$

for every point $x$ such that $\bar{B}(x, \varepsilon h) \subset \overline{c o n v}(P)$.

Proof. By optimality, $Z\left(x, \lambda^{*}(x)\right) \leq Z(x, 0)$ and, by Lemma $4.2, Z\left(x, \lambda^{*}(x)\right) \leq$ $c_{Z}=M_{Z}$ for every $x \in \operatorname{conv}(P)$. Since $P$ is an $h$-covering of $\overline{c o n v}(P)$, there exists a point $x_{0} \in P$ at distance to $x$ less than or equal to $h$. In addition, by Lemma 4.3, there exists a constant $c_{\lambda}$ such that $\left|\lambda^{*}(x)\right| \leq \frac{c_{\lambda}}{\varepsilon_{2} h}$, where $\varepsilon_{2}=\min \{\varepsilon, 1\}$. We thus have

$$
Z\left(x, \lambda^{*}(x)\right) \geq e^{-\beta\left|x-x_{0}\right|^{2}+\left\langle\lambda^{*}(x), x-x_{0}\right\rangle} \geq e^{-\gamma-\left|\lambda^{*}(x)\right|\left|x-x_{0}\right|} \geq e^{-\gamma-\frac{c_{\lambda}}{\varepsilon_{2}}}=m_{Z}>0,
$$

as advertised.

Recall that $J^{*}(x) \in \mathbb{R}^{d \times d}$ is the Hessian of $\log Z\left(x, \lambda^{*}(x)\right)$ with respect to $\lambda$, (4.10). We proceed to estimate $\left\|J^{*}(x)^{-1}\right\|$.

Lemma 4.5. Let $P$ be a point set that is an $h$-covering of $\Omega$ with h-density bounded by $\tau$ for some $h, \tau>0$. Let $\beta=\frac{\gamma}{h^{2}}$ for some $\gamma>0$. Let $\varepsilon>0$. Let $x$ be such that $\bar{B}(x, \varepsilon h) \subset \overline{c o n v}(P)$. Then, there exists a constant $c_{J^{-1}}>0$ that depends on $\tau, \gamma, \varepsilon$, and d such that

$$
\left\|J^{*}(x)^{-1}\right\| \equiv \sup _{y \neq 0} \frac{\left|J^{*}(x)^{-1}(y)\right|}{|y|} \leq c_{J^{-1}} h^{-2} .
$$

Proof. Let $u \neq 0$ be a fixed vector. Then, from (4.10) we have

$$
u^{T} J^{*}(x) u=\frac{\sum_{a \in I} e^{-\beta\left|x-x_{a}\right|^{2}+\left\langle\lambda^{*}(x), x-x_{a}\right\rangle}\left\langle u, x-x_{a}\right\rangle^{2}}{Z\left(x, \lambda^{*}(x)\right)} .
$$

Next, we analyze the numerator and denominator of the right-hand side in turn. Let $\varepsilon_{2}=\min \{\varepsilon, 1\}$. By Lemma 4.1, there exists a point $x_{u} \in P$ such that $\varepsilon_{2} h \leq\left|x-x_{u}\right| \leq$ $\left(\varepsilon_{2}+1\right) h$ and $\left\langle u, x-x_{u}\right\rangle \geq|u| \varepsilon_{2} h$. Since $\bar{B}(x, \varepsilon h) \subset \overline{c o n v}(P)$, by Lemma 4.3 there exists a constant $c_{\lambda}$ such that $\left|\lambda^{*}(x)\right| \leq \frac{c_{\lambda}}{\varepsilon_{2} h}$, and we have

$$
\left|\left\langle\lambda^{*}(x), x-x_{u}\right\rangle\right| \leq\left|\lambda^{*}(x)\right|\left|x-x_{u}\right| \leq \frac{c_{\lambda}}{\varepsilon_{2} h}\left(\varepsilon_{2}+1\right) h=\frac{c_{\lambda}}{\varepsilon_{2}}\left(\varepsilon_{2}+1\right) .
$$

Hence,

$$
\begin{aligned}
& \sum_{a \in I} e^{-\beta\left|x-x_{a}\right|^{2}+\left\langle\lambda^{*}(x), x-x_{a}\right\rangle}\left\langle u, x-x_{a}\right\rangle^{2} \\
& \quad \geq e^{-\beta\left|x-x_{u}\right|^{2}+\left\langle\lambda^{*}(x), x-x_{u}\right\rangle}\left\langle u, x-x_{u}\right\rangle^{2} \geq e^{-\left(\varepsilon_{2}+1\right)^{2} \gamma-\frac{c_{\lambda}}{\varepsilon_{2}}\left(\varepsilon_{2}+1\right)}|u|^{2} \varepsilon_{2}^{2} h^{2},
\end{aligned}
$$

Copyright (c) by SIAM. Unauthorized reproduction of this article is prohibited. 
where we write $\beta=\frac{\gamma}{h^{2}}$. Combining the bound supplied by Lemma 4.4 with (4.31), we get

$$
\left|u^{T} J^{*}(x) u\right| \geq e^{-\left(\varepsilon_{2}+1\right)^{2} \gamma-\frac{c_{\lambda}}{\varepsilon_{2}}\left(\varepsilon_{2}+1\right)} \frac{\varepsilon_{2}^{2}}{M_{Z}}|u|^{2} h^{2}=c_{J}|u|^{2} h^{2},
$$

where $c_{J}=e^{-\left(\varepsilon_{2}+1\right)^{2} \gamma-\frac{c_{\lambda}}{\varepsilon_{2}}\left(\varepsilon_{2}+1\right)} \frac{\varepsilon_{2}^{2}}{M_{2}}>0$ depends on $\gamma, \tau, \varepsilon$, and $d$ only. Let $\lambda_{\min }(x)$ be the smallest eigenvalue of $J^{*}(x)$. Since $J^{*}(x)$ is positive definite [2], it follows that $\lambda_{\min }(x)>0$. Inequality (4.32) then implies that $\lambda_{\min }(x) \geq c_{J} h^{2}$. Since $\left\|J^{*}(x)^{-1}\right\|=$ $1 / \lambda_{\min }(x)$, the estimate (4.28) follows immediately with $c_{J^{-1}}=1 / c_{J}$.

We are finally in a position to estimate the derivatives of the LME basis functions.

Proposition 4.6. Let $P$ be a point set that is an h-covering of $\Omega$ with h-density bounded by $\tau$ for some $h, \tau>0$. Let $\beta=\frac{\gamma}{h^{2}}$ for some $\gamma>0$ and $\varepsilon>0$. Let $W=\left\{w_{a}^{*}: a \in I\right\}$ be the optimal basis functions of the LME approximation scheme with node set $P$ and parameter $\beta$. Then,

$$
\left|\nabla w_{a}^{*}(x)\right| \leq c_{J^{-1}} w_{a}^{*}(x)\left|x-x_{a}\right| h^{-2}
$$

for every point $x$ such that $\bar{B}(x, \varepsilon h) \subset \overline{c o n v}(P)$ and every point $x_{a} \in P$.

Proof. The estimate (4.33) follows immediately from Lemma 4.5 and (4.11).

Next we show that the LME approximation scheme has polynomial decay of order $(1, s)$ for every $s \geq 1$.

Proposition 4.7. Let $\{I, W, P\}$ be an LME approximation scheme. Suppose that $P$ is an h-covering of $\Omega$, and $P$ has $h$-density bounded by $\tau, \beta=\gamma / h^{2}$ for some $\gamma>0$. Let $\varepsilon>0$ and $s \geq 1$. Then, there exists a constant $c>0$ (depending on $d, \gamma$, $\tau, \varepsilon$, and $s)$ such that the approximation scheme has polynomial decay of order $(1, s)$ for $c$ and $h$ in $\Omega_{\varepsilon h}=\left\{x \in \mathbb{R}^{d}\right.$ s.t. $\left.\bar{B}(x, \varepsilon h) \subset \overline{c o n v}(P)\right\}$.

Proof. We recall that the LME basis functions are $C^{\infty}$ on $\operatorname{conv}(P)$ (see [2]). Next, we show that there exists a constant $c>0$ that depends on $\gamma, \tau, d, \varepsilon$, and $s$ such that, for any $k$,

$$
\sup _{|\alpha| \leq 1} \sup _{x \in \Omega_{\varepsilon h}} \sup _{a \in I}\left(1+\left|\frac{x-x_{a}}{h}\right|^{2}\right)^{s} h^{|\alpha|}\left|D^{\alpha} w_{a}^{*}(x)\right| \leq c .
$$

From Lemmas 4.3 and 4.4 we have

$$
\begin{aligned}
0 & \leq w_{a}^{*}(x)=\frac{e^{-\beta\left|x-x_{a}\right|^{2}+\left\langle\lambda^{*}(x), x-x_{a}\right\rangle}}{Z\left(x, \lambda^{*}(x)\right)} \\
& \leq \frac{e^{-\gamma\left|\left(x-x_{a}\right) / h\right|^{2}+\left|\lambda^{*}(x)\right|\left|x-x_{a}\right|}}{m_{Z}} \leq \frac{e^{-\gamma\left|\left(x-x_{a}\right) / h\right|^{2}+\tilde{c}_{\lambda}\left|\left(x-x_{a}\right) / h\right|}}{m_{Z}},
\end{aligned}
$$

with $\tilde{c}_{\lambda}=c_{\lambda} / \min \{\varepsilon, 1\}$. In addition,

$$
\begin{aligned}
& \sup _{x \in \Omega_{\varepsilon h}} \sup _{a \in I}\left(1+\left|\frac{x-x_{a}}{h}\right|^{2}\right)^{s} w_{a}^{*}(x) \\
& \leq \sup _{x \in \Omega_{\varepsilon h}} \sup _{a \in I}\left(1+\left|\frac{x-x_{a}}{h}\right|^{2}\right)^{s} \frac{e^{-\gamma\left|\left(x-x_{a}\right) / h\right|^{2}+\tilde{c}_{\lambda}\left|\left(x-x_{a}\right) / h\right|}}{m_{Z}} \\
& \leq c^{\prime}:=\sup _{t \geq 0}\left(1+t^{2}\right)^{s} \frac{e^{-\gamma t^{2}+\tilde{c}_{\lambda} t}}{m_{Z}}<\infty
\end{aligned}
$$

Copyright $\odot$ by SIAM. Unauthorized reproduction of this article is prohibited. 
since $\frac{e^{-\gamma t^{2}+\tilde{c}_{\lambda} t}}{m_{Z}}$ is a rapidly decreasing function of $t$. We note that $c^{\prime}$ is defined in terms of the constants $d, \gamma, \tau, \varepsilon$, and $s$. Thus, by Proposition 4.6 we have

$$
\begin{aligned}
& \sup _{x \in \Omega_{\varepsilon h}} \sup _{a \in I}\left(1+\left|\frac{x-x_{a}}{h}\right|^{2}\right)^{s} h\left|\nabla w_{a}^{*}(x)\right| \\
& \leq \sup _{x \in \Omega_{\varepsilon h}} \sup _{a \in I}\left(1+\left|\frac{x-x_{a}}{h}\right|^{2}\right)^{s} h c_{J^{-1}} w_{a}^{*}(x)\left|x-x_{a}\right| h^{-2} \\
& \leq \sup _{x \in \Omega_{\varepsilon h}} \sup _{a \in I}\left(1+\left|\frac{x-x_{a}}{h}\right|^{2}\right)^{s} c_{J^{-1}} w_{a}^{*}(x)<c_{J^{-1}} c^{\prime},
\end{aligned}
$$

as advertised.

The next theorem bounds uniformly the error of the approximate function $u_{k}$ and its derivatives to a smooth function $u$ and its derivatives. The result is based on Theorem 3.5, which holds for a general approximation scheme.

TheOREm 4.8. Under the assumptions of Proposition 4.7, let $u \in C^{2}(\bar{\Omega})$. Then, there exists a constant $C>0$ that depends on $\gamma, \tau, \varepsilon$, and $d$ only such that

$$
\left|D^{\alpha} u_{I}(x)-D^{\alpha} u(x)\right| \leq C\left\|D^{2} u\right\|_{\infty} h^{2-|\alpha|}
$$

for $x \in \Omega_{\varepsilon h},|\alpha| \leq 1$

Proof. The theorem follows from Proposition 4.7 and Theorem 3.5.

Finally, we are in a position to show that LME approximation spaces on a domain $\Omega^{\prime}$ are dense in $W^{1, p}(\Omega)$ for subdomains $\Omega \subset \Omega^{\prime}$ which are compactly contained in $\Omega^{\prime}$. This result is derived from the polynomial decay of LME schemes and the density of approximation schemes of Corollary 3.7.

Corollary 4.9. Let $\Omega$ be a domain satisfying the segment condition, and let $\Omega^{\prime}$ be an auxiliary domain such that $\bar{\Omega} \subset \Omega^{\prime}$. Let $\left\{I_{k}, W_{k}, P_{k}\right\}$ be a sequence of LME approximation schemes in $\Omega^{\prime}$. Suppose that the assumptions of Proposition 4.7 hold for $\left\{I_{k}, W_{k}, P_{k}\right\}$ in $\Omega^{\prime}$ uniformly for $h_{k} \rightarrow 0$. Let $1 \leq p<\infty$. Then, for every $u \in W^{1, p}(\Omega)$, there exists a sequence $u_{k} \in X_{k}$ such that $u_{k \mid \Omega} \rightarrow u$.

Proof. As $\bar{\Omega} \subset \Omega^{\prime}$, there exists $r>0$ such that $\cup_{x \in \Omega} B(x, r)=\left\{x \in \mathbb{R}^{d}: \operatorname{dist}(x, \Omega)\right.$ $<r\} \subset \Omega^{\prime}$. The sequence of approximation schemes $\left\{I_{k}, W_{k}, P_{k}\right\}$, when restricted to $\Omega$, has uniform polynomial decay $(1, s)$ for any fixed $s$. Then, the theorem follows from Corollary 3.7.

Corollary 4.9 guarantees the density of the LME approximates on $W^{1, p}(\Omega)$ provided that the sequence of LME approximation schemes $\left\{I_{k}, W_{k}, P_{k}\right\}$ is defined on a bigger domain $\Omega^{\prime}$. We note that, in this case, the LME scheme does not obey the weak Kronecker-delta property at the boundary of $\Omega$, making it less straightforward to enforce boundary conditions on $\Omega$. However, imposing boundary conditions can be done in this case by using standard Lagrangian multipliers; see, e.g., [14, 9].

5. Application to the local maximum-entropy approximation scheme: Estimates up to the boundary. In section 4 we have seen that, for a sequence $\left\{I_{k}, W_{k}, P_{k}\right\}$ of LME approximation schemes, we have density of the approximation space $X_{k}$ in $W_{\text {loc }}^{1, p}(\Omega)$. In order to treat boundary value problems, however, we need density results up to the boundary of $\Omega$. A way to guarantee the density in $W^{1, p}(\Omega)$ is to work with a sequence $\left\{I_{k}, W_{k}, P_{k}\right\}$ defined on a (strictly) bigger domain $\Omega^{\prime}$, as discussed in Corollary 4.9. In this section, we analyze the density of the approximation space $X_{k}$ when the domain of the LME scheme is $\Omega$. 
While we will see that density can be extended to $W_{0}^{1, p}(\Omega)$ in general, a major technical difficulty with estimates up to the boundary comes from the fact that $\lambda^{*}(x)$ blows up as $x$ approaches $\partial \Omega$. This blowup is indeed a manifestation of the weak Kronecker-delta property at the boundary, as $\lambda^{*}$ will blowup in such a way that in the limit no weight is given to nodal data in the interior of $\Omega$. For general $\Omega$, this behavior can become very complicated and lead to blow up of the gradients of the optimal basis functions $\nabla w_{a}$, with the result that the general convergence scheme of section 3 is no longer applicable. Therefore, for simplicity we restrict our attention to the class of polyhedral domains. While an estimate as in (4.34) is not guaranteed to hold and $\left(1+\left|\frac{x-x_{a}}{h}\right|^{2}\right)^{s} h\left|\nabla w_{a}^{*}(x)\right|$ might blow up as $x \rightarrow \partial \Omega$, under generic assumptions we shall show that near flat pieces of the boundary the rate of blowup can be bounded by $C_{\mu}\left(1+h^{\mu} \operatorname{dist}^{-\mu}(x, \partial \Omega)\right)$ for any $\mu>0$; see Proposition 5.8. Such an estimate on $\nabla w^{*}$ then permits us to prove that, away from a small singular part of the boundary, Sobolev functions can be approximated by linear combinations of basis functions in the limit of $h \rightarrow 0$. The singular boundary is of finite 2-capacity. With the help of a capacity argument we can then establish approximation results with truncated LME functions in $H^{1}(\Omega)$ for spatial dimension $d>2$. It is worth noting that, once bounds on $\left|\nabla w_{a, h}^{*}\right|$ (as in Propositions 4.7 and 5.8 and Lemma 5.9) have been established, our further arguments are not restricted to LME approximations but rather also apply to general convergence schemes as discussed in section 3 .

More precisely, in this section we will assume that $\Omega$ is a convex polytope in $\mathbb{R}^{d}$ and $P$ is an $h$-covering for $\Omega$ with conv $P=\Omega$ such that there exists a constant $\eta>0$ such that $\{x \in P: 0<\operatorname{dist}(x, \partial \Omega)<\eta h\}=\emptyset$. Note that then $P \cap \partial \Omega$ is an $h$-covering for $\partial \Omega$.

Assume that $A=H \cap \partial \Omega$, where $H$ is some hyperplane, is a flat $(d-1)$-dimensional subset of the boundary of $\Omega$. With the aim of controlling $\nabla w^{*}(x)$ for $x$ in the vicinity of $A$, our first task will be to exactly estimate the behavior of $J^{*}(x)$ in this regime. First note that with a proper choice of the coordinate system we may assume that $H=\left\{x_{1}=0\right\}=\{0\} \times \mathbb{R}^{d-1}$ with $\Omega \cap\left\{x_{1} \geq 0\right\}=\Omega$. Accordingly, we write

$$
\lambda^{*}(x)=\left(\lambda_{1}^{*}(x), \lambda^{\prime}(x)\right) \in \mathbb{R} \times \mathbb{R}^{d-1}
$$

and, for $x=\left(x_{1}, x^{\prime}\right)$,

$$
Z=\sum_{a \in I} e^{-\beta\left|x-x_{a}\right|^{2}+\left\langle\lambda^{\prime}, x^{\prime}-x_{a}^{\prime}\right\rangle+\left(x-x_{a}\right)_{1} \lambda_{1}^{*}} .
$$

We fix $\delta>0$ and consider points $x \in \Omega$ with $x=\left(\rho, x^{\prime}\right) \in \mathbb{R} \times \mathbb{R}^{d-1}$ for $\rho$ small such that $B_{\delta h}\left(0, x^{\prime}\right) \cap H=B_{\delta h}\left(0, x^{\prime}\right) \cap A$. In the following lemmas we will also set $h=1$ for arbitrarily large $\Omega$ and recover the general case in Proposition 5.8 by rescaling afterward. Generic positive constants, denoted $c, c^{\prime}, c^{\prime \prime}$ or $C, C^{\prime}$, will be independent of $\rho$ and the size of $\Omega$.

Lemma 5.1. There is a constant $C>0$ such that

$$
\left|\lambda^{\prime}(x)\right| \leq C \quad \text { and } \quad \lambda_{1}^{*}(x) \geq-C .
$$

Proof. This result follows along the same lines as the proof of Lemma 4.3 for the boundedness of $\lambda^{*}$ in the interior of $\Omega$.

In order to investigate $Z$ we split the sum as

$$
Z=\sum_{a \in I_{A}} e^{-\beta\left|x-x_{a}\right|^{2}+\left\langle\lambda^{\prime}, x^{\prime}-x_{a}^{\prime}\right\rangle+\rho \lambda_{1}^{*}}+\sum_{a \in I \backslash I_{A}} e^{-\beta\left|x-x_{a}\right|^{2}+\left\langle\lambda^{\prime}, x^{\prime}-x_{a}^{\prime}\right\rangle-\left(x_{a}-x\right)_{1} \lambda_{1}^{*}},
$$

where $I_{A}$ collects those indices $a$ for which $x_{a} \in A$. 
Lemma 5.2. As $\rho$ tends to $0, \lambda_{1}^{*} \rightarrow \infty$ such that $\rho \lambda_{1}^{*} \rightarrow 0$.

Proof. Writing $Z$ as in (5.4) and noting that $\left(x_{a}-x\right)_{1}>\eta$ for $a \notin I_{A}$ and $\rho>0$, we see that

$$
Z \geq \sum_{a \in I_{A}} e^{-\beta\left|x-x_{a}\right|^{2}+\left\langle\lambda^{\prime}, x^{\prime}-x_{a}^{\prime}\right\rangle}
$$

and that this lower bound is in fact achieved only if $\rho \lambda_{1}^{*} \rightarrow 0$ and $\lambda_{1}^{*} \rightarrow \infty$.

In particular, we see that $Z$ still remains bounded from above and from below by positive constants. implies

In order to estimate $J^{*}$, we first observe that the optimality condition $\frac{\partial Z}{\partial \lambda_{1}}=0$

$$
\rho \sum_{a \in I_{A}} e^{-\beta\left|x-x_{a}\right|^{2}\left\langle\lambda^{\prime}, x^{\prime}-x_{a}^{\prime}\right\rangle+\rho \lambda_{1}^{*}}=\sum_{a \in I \backslash I_{A}} e^{-\beta\left|x-x_{a}\right|^{2}+\left\langle\lambda^{\prime}, x^{\prime}-x_{a}^{\prime}\right\rangle-\left(x_{a}-x\right)_{1} \lambda_{1}^{*}}\left(x_{a}-x\right)_{1} .
$$

Lemma 5.3. There is a constant $c>0$ such that the first entry $J_{11}^{*}$ in $J^{*}(x)$ satisfies

$$
J_{11}^{*} \geq c \rho .
$$

Proof. Since $\left(x_{a}-x\right)_{1} \geq \eta$ for $a \notin I_{A}$, we find by (5.6) and Lemma 5.1 that

$$
\begin{aligned}
J_{11}^{*} & =\sum_{a \in I} w_{a}^{*}(x)\left(x-x_{a}\right)_{1}^{2} \\
& \geq Z^{-1} \sum_{a \in I \backslash I_{A}} e^{-\beta\left|x-x_{a}\right|^{2}+\left\langle\lambda^{\prime}, x^{\prime}-x_{a}^{\prime}\right\rangle-\left(x_{a}-x\right)_{1} \lambda_{1}^{*}}\left(x_{a}-x\right)_{1}^{2} \\
& \geq \eta Z^{-1} \sum_{a \in I \backslash I_{A}} e^{-\beta\left|x-x_{a}\right|^{2}+\left\langle\lambda^{\prime}, x^{\prime}-x_{a}^{\prime}\right\rangle-\left(x_{a}-x\right)_{1} \lambda_{1}^{*}}\left(x_{a}-x\right)_{1} \\
& =\eta \rho Z^{-1} \sum_{a \in I_{A}} e^{-\beta\left|x-x_{a}\right|^{2}+\left\langle\lambda^{\prime}, x^{\prime}-x_{a}^{\prime}\right\rangle+\rho \lambda_{1}^{*}} \\
& \geq c \rho
\end{aligned}
$$

for some constant $c>0$.

We now derive an upper bound for the entries of the first row and column of $J^{*}$.

Lemma 5.4. For any $0<\mu<1$, there exists a constant $C>0$ such that

$$
\left|J_{1 j}^{*}\right|=\left|J_{j 1}^{*}\right| \leq C \rho^{\mu}, \quad j=1, \ldots, d .
$$

For $j=1, \ldots, d$ we have $J_{1 j}^{*}=J_{j 1}^{*}=\sum_{a \in I} w_{a}^{*}(x)\left(x-x_{a}\right)_{1}\left(x-x_{a}\right)_{j}$. First, summing over $a \in I_{A}$ gives the obvious bound

$$
\begin{aligned}
\left|\sum_{a \in I_{A}} w_{a}^{*}(x)\left(x-x_{a}\right)_{1}\left(x-x_{a}\right)_{j}\right| & \leq Z^{-1} \sum_{a \in I_{A}} e^{-\beta\left|x-x_{a}\right|^{2}+\left\langle\lambda^{\prime}, x^{\prime}-x_{a}^{\prime}\right\rangle+\rho \lambda_{1}^{*}} \rho\left|x-x_{a}\right| \\
& \leq C \rho .
\end{aligned}
$$

Copyright $@$ by SIAM. Unauthorized reproduction of this article is prohibited. 
In order to estimate the remaining sum, we let $p=\frac{1}{\mu}$ and choose $1<q<\infty$ with $\frac{1}{p}+\frac{1}{q}=1$ so that

$$
\begin{aligned}
& \left|\sum_{a \in I \backslash I_{A}} w_{a}^{*}(x)\left(x-x_{a}\right)_{1}\left(x-x_{a}\right)_{j}\right| \\
& \quad \leq\left(\sum_{a \in I \backslash I_{A}} w_{a}^{*}(x)\left(x_{a}-x\right)_{1}^{p}\right)^{\frac{1}{p}}\left(\sum_{a \in I \backslash I_{A}} w_{a}^{*}(x)\left|x-x_{a}\right|_{j}^{q}\right)^{\frac{1}{q}}
\end{aligned}
$$

by Hölder's inequality. Here the second factor in (5.11) is bounded by

$$
\left(Z^{-1} \sum_{a \in I \backslash I_{A}} e^{-\beta\left|x-x_{a}\right|^{2}+\left\langle\lambda^{\prime}, x^{\prime}-x_{a}^{\prime}\right\rangle-\left(x_{a}-x\right)_{1} \lambda_{1}^{*}}\left|x-x_{a}\right|_{j}^{q}\right)^{\frac{1}{q}} \leq C ;
$$

see Lemma 5.1. To estimate the first factor we note that, since $P$ is a 1-covering of $\Omega$, there exists $\bar{a} \in I \backslash I_{A}$ such that

$$
\left|x_{\bar{a}}-x\right| \leq C^{\prime}
$$

for a constant $C^{\prime}>\eta$. For $\rho$ sufficiently small and thus $\lambda_{1}^{*}$ sufficiently large, we then have the estimate

$$
\begin{aligned}
\sum_{\substack{a \in I \backslash I_{A} \\
\left(x_{a}-x\right)_{1} \geq C^{\prime}}} w_{a}^{*}(x)\left(x_{a}-x\right)_{1}^{p} & \leq Z^{-1} \sum_{\substack{a \in I \backslash I_{A} \\
\left(x_{a}-x\right)_{1} \geq C^{\prime}}} e^{-\beta\left|x-x_{a}\right|^{2}+\left\langle\lambda^{\prime}, x^{\prime}-x_{a}^{\prime}\right\rangle-C^{\prime} \lambda_{1}^{*}}\left(x_{a}-x\right)_{1}^{p} \\
& \leq C Z^{-1} e^{-C^{\prime} \lambda_{1}^{*}} \\
& \leq C Z^{-1} e^{-\beta\left|x-x_{\bar{a}}\right|^{2}+\left\langle\lambda^{\prime}, x^{\prime}-x_{\bar{a}}^{\prime}\right\rangle-\left(x_{\bar{a}}-x\right)_{1} \lambda_{1}^{*}} \\
& \leq C Z^{-1} \eta^{-1} \sum_{a \in I \backslash I_{A}} e^{-\beta\left|x-x_{a}\right|^{2}+\left\langle\lambda^{*}, x-x_{a}\right\rangle}\left(x_{a}-x\right)_{1},
\end{aligned}
$$

as $\left(x_{a}-x\right)_{1} \geq \eta$ for $a \in I \backslash I_{A}$. On the other hand, for $a$ with $\left(x_{a}-x\right)_{1} \leq C^{\prime}$ we have the bound

$$
\left(x_{a}-x\right)_{1}^{p} \leq C\left(x_{a}-x\right)_{1} .
$$

Combining the two last estimates, we see that the term in the first factor of (5.11) satisfies

$$
\sum_{a \in I \backslash I_{A}} w_{a}^{*}(x)\left(x-x_{a}\right)_{1}^{p} \leq C \sum_{a \in I \backslash I_{A}} w_{a}^{*}(x)\left(x-x_{a}\right)_{1} .
$$

Since by (5.6) this last expression is bounded by $C \rho$, we arrive at

$$
\left|\sum_{a \in I \backslash I_{A}} w_{a}^{*}(x)\left(x-x_{a}\right)_{1}\left(x-x_{a}\right)_{j}\right| \leq C \rho^{\frac{1}{p}}=C \rho^{\mu}
$$

Copyright $@$ by SIAM. Unauthorized reproduction of this article is prohibited. 
by (5.11). Together with the bound (5.10) for the first part of the sum we have shown that indeed

$$
\left|J_{1 i}^{*}\right| \leq C \rho^{\mu}
$$

For the remaining part $B=\left(J_{i j}^{*}\right)_{2 \leq i, j \leq n}$ of the matrix $J^{*}$ we obtain the following lower matrix bound.

LEMMA 5.5. There is a constant $c>0$ such that

$$
B \geq c \operatorname{Id}_{n-1} .
$$

Proof. As $P \cap H$ is a 1 -covering for $A$, there is a set $J=\left\{a_{1}, \ldots, a_{d-1}\right\} \subset I_{A}$ of $d-1$ points such that $c^{\prime} \leq\left|x^{\prime}-x_{a}^{\prime}\right| \leq c^{\prime \prime}$ and $\operatorname{det}\left(x^{\prime}-x_{a_{1}}^{\prime}, \ldots, x^{\prime}-x_{a_{d-1}}^{\prime}\right) \geq c^{\prime}$ for suitable constants $c^{\prime}$ and $c^{\prime \prime}$. Then

$$
\begin{aligned}
B & =\sum_{a \in I} w_{a}^{*}(x)\left(x^{\prime}-x_{a}^{\prime}\right) \otimes\left(x^{\prime}-x_{a}^{\prime}\right) \\
& \geq Z^{-1} \sum_{a \in J} e^{-\beta\left|x-x_{\bar{a}}\right|^{2}+\left\langle\lambda^{\prime}, x^{\prime}-x_{\bar{a}}^{\prime}\right\rangle+\rho \lambda_{1}^{*}}\left(x^{\prime}-x_{a}^{\prime}\right) \otimes\left(x^{\prime}-x_{a}^{\prime}\right) \\
& \geq c \sum_{a \in J}\left(x^{\prime}-x_{a}^{\prime}\right) \otimes\left(x^{\prime}-x_{a}^{\prime}\right) \\
& \geq c \operatorname{Id}_{d-1}
\end{aligned}
$$

since all the projections $\left(x^{\prime}-x_{a}^{\prime}\right) \otimes\left(x^{\prime}-x_{a}^{\prime}\right)$ are nonnegative.

As a consequence of the above results, we obtain an estimate for the inverse matrix $\left(J^{*}\right)^{-1}=\left(\tilde{J}_{i j}\right)$.

Lemma 5.6. For any $0<\mu<\frac{1}{2}$ there exists a constant $C$ such that

$$
\left|\tilde{J}_{i j}\right| \begin{cases}\leq C \rho^{-1} & \text { for } i=j=1, \\ \leq C \rho^{-\mu} & \text { for } i=1, j=2, \ldots, d \text { or } j=1, i=2, \ldots, d, \text { and } \\ \leq C & \text { for } i, j=2, \ldots, d .\end{cases}
$$

Proof. First note that, expanding with respect to the first row, for $\frac{1}{2}<\tilde{\mu}<1$ we have

$$
\operatorname{det} J^{*}=J_{11}^{*} \operatorname{det} B+O\left(\rho^{2 \tilde{\mu}}\right)=J_{11}^{*} \operatorname{det} B \geq c J_{11}^{*} \geq c \rho
$$

by Lemmas $5.3,5.4$, and 5.5. Furthermore, as $\left|J^{*}\right| \leq C$, we have

$$
\left|\left(\operatorname{cof} J^{*}\right)_{i j}\right| \begin{cases}=|\operatorname{det} B| \leq C & \text { for } i=j=1 \\ \leq C \rho^{\tilde{\mu}} & \text { for } i=1, j \geq 2 \text { or } j=1, i \geq 2, \text { and } \\ \leq C\left(J_{11}^{*}+\rho^{2 \tilde{\mu}}\right) & \text { for } i, j \geq 2\end{cases}
$$

for $C$ sufficiently large. Now, Cramer's rule

$$
\left(J^{*}\right)^{-1}=\left(\operatorname{det} J^{*}\right)^{-1}\left(\operatorname{cof} J^{*}\right)^{T}
$$

implies

$$
\left|\tilde{J}_{i j}\right| \begin{cases}\leq C \rho^{-1} & \text { for } i=j=1, \\ \leq C \rho^{\tilde{\mu}-1} & \text { for } i=1, j \geq 2 \text { or } j=1, i \geq 2, \text { and } \\ \leq C & \text { for } i, j \geq 2,\end{cases}
$$

and thus the assertion follows by choosing $\tilde{\mu}$ such that $\mu=1-\tilde{\mu}$. 
Lemma 5.7. For any $s>0$ and $0<\mu<\frac{1}{2}$ there is a constant $C>0$ such that

$$
\left(1+\left|x-x_{a}\right|^{2}\right)^{s}\left|\nabla w_{a}^{*}(x)\right| \leq C\left(1+\rho^{-\mu}\left|x-x_{a}\right|\right) .
$$

Proof. If $a \in I_{A}$, then $x-x_{a}=\left(\rho, x^{\prime}-x_{a}^{\prime}\right)$, and Lemma 5.6 shows

$$
\left|\left(J^{*}\right)^{-1}\left(x-x_{a}\right)\right| \leq C\left(1+\rho^{-\mu}\left|x^{\prime}-x_{a}^{\prime}\right|\right) \leq C\left(1+\rho^{-\mu}\left|x-x_{a}\right|\right) .
$$

So, by (4.11),

$$
\left|\nabla w_{a}^{*}(x)\right| \leq C\left|w_{a}^{*}(x)\right|\left(1+\rho^{-\mu}\left|x-x_{a}\right|\right) .
$$

Now, using that $\left(1+\left|x-x_{a}\right|^{2}\right)^{s}\left|w_{a}^{*}(x)\right| \leq C$ for any $a$, we see that the estimate holds true for $a \in I_{A}$.

On the other hand, if $a \notin I_{A}$, then Lemma 5.6 gives only

$$
\left|\left(J^{*}\right)^{-1}\left(x-x_{a}\right)\right| \leq C \rho^{-1}\left|x-x_{a}\right|,
$$

whence

$$
\left|\nabla w_{a}^{*}(x)\right| \leq C\left|w_{a}^{*}(x)\right| \rho^{-1}\left|x-x_{a}\right| .
$$

But since $\left(x_{b}-x\right)_{1} \geq \eta$ for all $b \notin I_{A}$, we also get

$$
\begin{aligned}
& \left(1+\left|x-x_{a}\right|^{2}\right)^{s}\left|w_{a}^{*}(x)\right| \\
& =\left(1+\left|x-x_{a}\right|^{2}\right)^{s} Z^{-1} e^{-\beta\left|x-x_{a}\right|^{2}+\left\langle\lambda^{\prime}, x^{\prime}-x_{a}^{\prime}\right\rangle-\left(x_{a}-x\right)_{1} \lambda_{1}^{*}} \\
& \leq Z^{-1} \eta^{-1} \sum_{b \in I \backslash I_{A}} e^{-\beta\left|x-x_{b}\right|^{2}+\left\langle\lambda^{\prime}, x^{\prime}-x_{b}^{\prime}\right\rangle-\left(x_{b}-x\right)_{1} \lambda_{1}^{*}}\left(x_{b}-x\right)_{1}\left(1+\left|x-x_{b}\right|^{2}\right)^{s} .
\end{aligned}
$$

This term can now be estimated by $C \rho^{\tilde{\mu}} \rho^{-1}$ for $0<\tilde{\mu}<1$ precisely as the left-hand side of (5.11) in Lemma 5.4, which leads to

$$
\left(1+\left|x-x_{a}\right|^{2}\right)^{s}\left|\nabla w_{a}^{*}(x)\right| \leq C \rho^{-\mu}\left|x-x_{a}\right|
$$

for $0<\mu<1$.

Undoing the rescaling of $h$, we can now summarize the previous lemmas in the following proposition on the boundary behavior of $\nabla w_{a}^{*}$ near flat parts of $\partial \Omega$.

Proposition 5.8. Suppose that $x=\left(\rho, x^{\prime}\right) \in \mathbb{R} \times \mathbb{R}^{d-1}$ is such that $B_{\delta h}\left(0, x^{\prime}\right) \cap$ $H=B_{\delta h}\left(0, x^{\prime}\right) \cap A$ for a boundary $(d-1)$-face $A=\partial \Omega \cap H$. Let $s>0$ and $0<\mu<\frac{1}{2}$. There is a constant $C>0$ such that

$$
\left(1+\left|\frac{x-x_{a}}{h}\right|^{2}\right)^{s} h\left|\nabla w_{a, h}^{*}(x)\right| \leq C\left(1+h^{\mu} \operatorname{dist}^{-\mu}(x, \partial \Omega)\right) .
$$

If $P$ is an $h$-covering for $\Omega$, then $h^{-1} P$ is a 1 -covering for $h^{-1} \Omega$. Using subscripts $h$ to highlight the dependence on $h$, we have

$$
\begin{aligned}
Z_{h}(x) & =\sum_{x_{b} \in P} e^{-\frac{\gamma}{h^{2}}\left|x-x_{b}\right|^{2}+\left\langle\lambda_{(h)}^{*}, x-x_{b}\right\rangle} \\
& =\sum_{x_{b} \in P} e^{-\gamma\left|\frac{x-x_{b}}{h}\right|^{2}+\left\langle h \lambda_{(h)}^{*}, \frac{x-x_{b}}{h}\right\rangle} \\
& =\sum_{x_{b} \in h^{-1} P} e^{-\gamma\left|\frac{x}{h}-x_{b}\right|^{2}+\left\langle h \lambda_{(h)}^{*}, \frac{x}{h}-x_{b}\right\rangle} .
\end{aligned}
$$

Copyright $@$ by SIAM. Unauthorized reproduction of this article is prohibited. 
This expression is minimized at $h \lambda_{(h)}^{*}(x)=\lambda_{(1)}^{*}\left(\frac{x}{h}\right)$. For the basis functions $w_{a, h}^{*}$ we denote by $w_{a, 1}$ the basis function corresponding to the node $\frac{x_{a}}{h} \in h^{-1} P$ and obtain

$$
\begin{aligned}
w_{a, h}^{*}(x) & =\frac{e^{-\frac{\gamma}{h^{2}}\left|x-x_{a}\right|^{2}+\left\langle\lambda_{(h)}^{*}, x-x_{a}\right\rangle}}{\sum_{x_{b} \in P} e^{-\frac{\gamma}{h^{2}}\left|x-x_{b}\right|^{2}+\left\langle\lambda_{(h)}^{*}, x-x_{b}\right\rangle}} \\
& =\frac{e^{-\gamma\left|\frac{x-x_{a}}{h}\right|^{2}+\left\langle h \lambda_{(h)}^{*}, \frac{x-x_{a}}{h}\right\rangle}}{\sum_{x_{b} \in P} e^{-\gamma\left|\frac{x-x_{b}}{h}\right|^{2}+\left\langle h \lambda_{(h)}^{*}, \frac{x-x_{b}}{h}\right\rangle}} \\
& =\frac{e^{-\gamma\left|\frac{x}{h}-\frac{x_{a}}{h}\right|^{2}+\left\langle\lambda_{(1)}^{*}\left(\frac{x}{h}\right), \frac{x}{h}-\frac{x_{a}}{h}\right\rangle}}{\sum_{x_{b} \in h^{-1} P} e^{-\gamma\left|\frac{x}{h}-x_{b}\right|^{2}+\left\langle\lambda_{(1)}^{*}\left(\frac{x}{h}\right), \frac{x}{h}-x_{b}\right\rangle}} \\
& =w_{a, 1}^{*}\left(\frac{x}{h}\right) .
\end{aligned}
$$

Applying Lemma 5.7 to $w_{a, 1}^{*}$, we therefore obtain

$$
\begin{aligned}
\left(1+\left|\frac{x-x_{a}}{h}\right|^{2}\right)^{s}\left|\nabla w_{a, h}^{*}(x)\right| & =\left(1+\left|\frac{x-x_{a}}{h}\right|^{2}\right)^{s} h^{-1}\left|\nabla w_{a, 1}^{*}\left(\frac{x}{h}\right)\right| \\
& \leq C h^{-1}\left(1+\operatorname{dist}^{-\mu}\left(\frac{x}{h}, A\right)\left|\frac{x}{h}-\frac{x_{a}}{h}\right|\right) .
\end{aligned}
$$

From our previous calculations we already know that the left-hand side is bounded by a constant away from the boundary of $\Omega$. Suppose that $x$ is such that $\operatorname{dist}^{-\mu}\left(\frac{x}{h}, A\right) \geq 1$. Since $s$ is arbitrary we can absorb the last factor on the right-hand side into the prefactor of the left-hand side and finally obtain

$$
\left(1+\left|\frac{x-x_{a}}{h}\right|^{2}\right)^{s} h\left|\nabla w_{a, h}^{*}(x)\right| \leq C\left(1+h^{\mu} \operatorname{dist}^{-\mu}(x, \partial \Omega)\right) .
$$

Now suppose that $x$ is a general point near a possibly lower dimensional edge of $\partial \Omega$. More precisely, $x$ is close to an $m$-face of $A$ of $\partial \Omega$, which is the intersection of $d-m$ hyperplanes $H_{1}, \ldots, H_{d-m}$ with linearly independent normals which constitute $\partial \Omega$ in the vicinity of $x$.

Lemma 5.9. There exists $R>0$ such that for all $x_{a} \in P$ with $\operatorname{dist}\left(x_{a}, \partial \Omega\right) \geq R h$

$$
\left(1+\left|\frac{x-x_{a}}{h}\right|^{2}\right)^{s} h\left|\nabla w_{a, h}^{*}(x)\right| \leq 1 .
$$

Proof. We first assume again that $h=1$. Let $H$ be the hyperplane containing $x$ which is perpendicular to $\lambda^{*}$. Similarly as in Lemma 5.1 we see that, as $\operatorname{dist}(x, A) \rightarrow 0$, $\left|\lambda^{*}\right|$ tends to infinity such that the projection of $\lambda^{*}$ onto $\bigcap H_{i}$ remains bounded and that there are constants $c, C>0$ such that

$$
\left\langle y-x, \frac{\lambda^{*}}{\left|\lambda^{*}\right|}\right\rangle=\operatorname{dist}(y-x, H) \geq c \operatorname{dist}\left(y, H_{1} \cup \ldots \cup H_{d-m}\right)-C
$$

for every $y \in \Omega$.

Choose a set $J=\left\{a_{1}, \ldots, a_{d}\right\} \subset I$ of $d$ points such that $c^{\prime} \leq\left|x-x_{a}\right| \leq c^{\prime \prime}$ and $\operatorname{det}\left(x-x_{a_{1}}, \ldots, x-x_{a_{d}}\right) \geq c^{\prime}$ for suitable constants $c^{\prime}$ and $c^{\prime \prime}$. Then

$$
J^{*} \geq Z^{-1} \sum_{a \in J} e^{-\beta\left|x-x_{a}\right|^{2}+\left\langle\lambda^{*}, x-x_{a}\right\rangle}\left(x-x_{a}\right) \otimes\left(x-x_{a}\right) \geq c e^{-C\left|\lambda^{*}\right|} \operatorname{Id}_{d-1}
$$

Copyright $@$ by SIAM. Unauthorized reproduction of this article is prohibited. 
since all the projections $\left(x-x_{a}\right) \otimes\left(x-x_{a}\right)$ are nonnegative. It follows that

$$
\left(J^{*}\right)^{-1} \leq C e^{C\left|\lambda^{*}\right|} \operatorname{Id}_{d-1} .
$$

Now if $x_{a} \in \Omega$ satisfies $\operatorname{dist}(x, \partial \Omega) \geq R$, then

$$
\left\langle x-x_{a}, \lambda^{*}\right\rangle \leq-\left(c \operatorname{dist}\left(x_{a}, H_{1} \cup \ldots \cup H_{d-m}\right)+C\right)\left|\lambda^{*}\right| \leq(-c R+C)\left|\lambda^{*}\right| .
$$

So

$$
\left|w_{a}^{*}\right| \leq Z^{-1} e^{-\beta\left|x-x_{a}\right|^{2}+\left\langle\lambda^{*}, x-x_{a}\right\rangle} \leq Z^{-1} e^{-\beta\left|x-x_{a}\right|^{2}-(c R-C)\left|\lambda^{*}\right|} .
$$

It follows from (4.11) that

$$
\begin{aligned}
\left(1+\left|x-x_{a}\right|^{2}\right)^{s}\left|\nabla w_{a}^{*}(x)\right| & \leq C\left(1+\left|x-x_{a}\right|^{2}\right)^{s} e^{-\beta\left|x-x_{a}\right|^{2}-(c R+C)\left|\lambda^{*}\right|} e^{C\left|\lambda^{*}\right|}\left|x-x_{a}\right| \\
& \leq C e^{(2 C-c R)\left|\lambda^{*}\right|} \leq 1
\end{aligned}
$$

for $R$ sufficiently large, which proves the lemma for $h=1$. The estimate for general $h$ now follows directly by rescaling as before.

We are now in a position to prove our main density results up to the boundary. Density in $W_{0}^{1, p}(\Omega)$ in fact relies only on our previous interior estimates (see section 4 and Lemma 5.9) and is true for general, not necessarily polyhedral domains $\Omega$.

THEOREM 5.10. Let $\Omega$ be a bounded polyhedron and let $\left\{I_{k}, W_{k}, P_{k}\right\}$ be a sequence of LME approximation schemes satisfying the assumptions of Proposition 4.7 uniformly for $h_{k} \rightarrow 0$. Then for any $u \in W_{0}^{1, p}(\Omega), 1 \leq p<\infty$, there exists a sequence $u_{k} \in X_{k}$ such that $u_{k} \rightarrow u$. we have

Proof. It suffices for us to consider $u \in C_{c}^{\infty}(\Omega)$. Let $u_{k}=u_{I_{k}}$. By Proposition 3.2

$$
\left|D^{\alpha} u_{k}(x)-D^{\alpha} u(x)\right| \leq \sum_{a \in I}\left|R_{2}\left(x_{a}, x\right)\right| D^{\alpha} w_{a}^{*}(x) \mid,
$$

which for $\operatorname{dist}(x, \partial \Omega) \geq \varepsilon h$ can be estimated by $C\left\|D^{2} u\right\|_{\infty} h_{k}^{2-|\alpha|} \rightarrow 0$ as $h_{k} \rightarrow 0$; see Theorem 3.5.

If $\operatorname{dist}(x, \partial \Omega) \leq \varepsilon h$, then with the help of Lemma 5.9 , precisely the same arguments as in the proof of Theorem 3.5 show that

$$
\sum_{\substack{a \in I \\\left|x-x_{a}\right| \geq R h}}\left|R_{2}\left(x_{a}, x\right)\right| D^{\alpha} w_{a}^{*}(x) \mid \leq C\left\|D^{2} u\right\|_{\infty} h_{k}^{2-|\alpha|} \rightarrow 0
$$

for a sufficiently large constant $R>0$. But the remaining part of the sum vanishes for small $h_{k}$ as $R_{2}\left(x_{a}, x\right)=0$ if $\left|x-x_{a}\right|<R h$, since then $u$ vanishes on a neighborhood of the segment $\left\{x_{a}+\theta\left(x-x_{a}\right): \theta \in[0,1]\right\} \subset\left\{x \in \Omega: \operatorname{dist}(x, \partial \Omega) \leq(R+\varepsilon) h_{k}\right\}$.

In order to formulate our main result on density up to the boundary we denote by $\partial_{*} \Omega$ the union of the interiors of the $(d-1)$-faces of $\partial \Omega$. $\left(\partial_{*} \Omega\right.$ is the reduced boundary in the language of geometric measure theory.) The part of $\Omega$ a distance $\varepsilon h$ away from the singular boundary $\partial \Omega \backslash \partial_{*} \Omega$ is denoted $\tilde{\Omega}_{\varepsilon h}=\left\{x \in \Omega\right.$ : $\left.\operatorname{dist}\left(x, \partial \Omega \backslash \partial_{*} \Omega\right) \geq \varepsilon h\right\}$.

THEOREM 5.11. Let $\Omega$ be a bounded polyhedron and let $\left\{I_{k}, W_{k}, P_{k}\right\}$ be a sequence of LME approximation schemes satisfying the assumptions of Proposition 4.7

Copyright $@$ by SIAM. Unauthorized reproduction of this article is prohibited. 
uniformly for $h_{k} \rightarrow 0$. Let $\varepsilon>0$. Then for any $u \in W^{1, p}(\Omega), 1 \leq p<\infty$, there exists a sequence $u_{k} \in X_{k}$ such that $\left\|u_{k}-u\right\|_{W^{1, p}\left(\tilde{\Omega}_{s h}\right)} \rightarrow 0$.

Therefore, density holds away from the singular boundary.

Proof. Let $u \in C^{2}(\Omega)$. As in the proof of Theorem 5.10 we find by Proposition 3.2 and the arguments in the proof of Theorem 3.5 that for all $x \in \tilde{\Omega}_{\varepsilon h}$

$$
\left|D^{\alpha} u_{I}(x)-D^{\alpha} u(x)\right| \leq C\left\|D^{2} u\right\|_{\infty} h^{2-|\alpha|}\left(1+h^{\mu} \operatorname{dist}^{-\mu}(x, \partial \Omega)\right),
$$

$0<\mu<\frac{1}{2}$, where in addition we have applied Proposition 5.8 in order to estimate $\nabla w_{a}^{*}$ near the regular boundary. Consequently,

$$
\begin{aligned}
\int_{\tilde{\Omega}_{\varepsilon h}}\left|D^{\alpha} u_{I}(x)-D^{\alpha} u(x)\right|^{p} d x & \leq C h^{(2-|\alpha|) p} \int_{\tilde{\Omega}_{\varepsilon h}} 1+h^{p \mu} \operatorname{dist}^{-p \mu}(x, \partial \Omega) d x \\
& \leq C h^{(2-|\alpha|) p}\left(1+h^{p \mu} \int_{0}^{1} t^{-p \mu} d t\right) \\
& =C h^{(2-|\alpha|) p}
\end{aligned}
$$

for $\mu$ sufficiently close to 0 .

Note that since the $(d-2)$-dimensional Hausdorff measure of $\partial \Omega \backslash \partial_{*} \Omega$ is finite, this set has zero 2-capacity for $d \geq 3$. Theorem 5.11 thus shows that $u$ can be approximated by $u_{k} \in X_{k}$ in $H^{1}$ up to sets of arbitrarily small 2-capacity with the help of a capacity argument we obtain therein.

Corollary 5.12. Let $\Omega$ be a bounded polyhedron and let $\left\{I_{k}, W_{k}, P_{k}\right\}$ be a sequence of LME approximation schemes, $\varepsilon>0$. Suppose that $d>2$. Then for any $u \in H^{1}(\Omega)$ there exist a sequence $\chi_{k} \in H^{1}$ with $\chi_{k} \rightarrow 1$ in $H^{1}$ and a sequence $u_{k} \in X_{k}$ such that $\chi_{k} u_{k} \rightarrow u$ in $H^{1}$.

Proof. Since the $(d-2)$-dimensional Hausdorff measure of the singular part $\partial \Omega \backslash \partial_{*} \Omega$ of the boundary is finite, this set has zero 2-capacity:

$$
\mathrm{Cap}_{2}\left(\partial \Omega \backslash \partial_{*} \Omega\right)=0 \text {. }
$$

In particular, for every neighborhood $V$ of $\partial \Omega \backslash \partial_{*} \Omega$ and $\delta>0$ there exists a function $\psi_{\delta} \in H^{1}\left(\mathbb{R}^{d}\right)$ with compact support in $V$ such that $\psi_{\delta}>1$ in a smaller neighborhood of $\partial \Omega \backslash \partial_{*} \Omega$ and

$$
\int_{\mathbb{R}^{d}}\left|\nabla \psi_{\delta}\right|^{2} d x<\delta
$$

(This follows, e.g., from Theorem 3 and its proof in [12, pp. 155-157].) By replacing, if necessary, $\psi_{\delta}$ with a mollification of $\max \left\{\min \left\{\psi_{\delta}, 1\right\},-1\right\}$ we may assume that $\psi_{\delta}$ is smooth, $\left|\psi_{\delta}\right| \leq 1$, and in particular $\psi_{\delta} \equiv 1$ near $\partial \Omega \backslash \partial_{*} \Omega$.

Now suppose that $u \in C^{1}(\bar{\Omega})$. By Theorem 5.11 we find $u_{k} \in X_{k}$ with $\| u_{k}-$ $u \|_{W^{1, p}\left(\tilde{\Omega}_{h_{k}}\right)} \rightarrow 0$ for all $p<\infty$. Since $1-\psi_{\delta}$ vanishes in a neighborhood of $\partial \Omega \backslash \partial_{*} \Omega$, it follows that

$$
\begin{aligned}
& \left\|\left(1-\psi_{\delta}\right)\left(u_{k}-u\right)\right\|_{H^{1}(\Omega)}^{2} \\
& \leq\left\|1-\psi_{\delta}\right\|_{L^{\infty}(\Omega)}^{2}\left\|u_{k}-u\right\|_{H^{1}\left(\tilde{\Omega}_{h_{k}}\right)}^{2}+\left\|\nabla \psi_{\delta}\right\|_{L^{2}(\Omega)}\left\|u_{k}-u\right\|_{L^{\infty}\left(\tilde{\Omega}_{h_{k}}\right)}^{2} \rightarrow 0
\end{aligned}
$$

by Sobolev embedding. As

$$
\begin{aligned}
\left\|\left(1-\psi_{\delta}\right) u-u\right\|_{H^{1}}^{2} & =\left\|\psi_{\delta} u\right\|_{H^{1}}^{2} \\
& \leq \int_{V}|u|^{2} d x+\int_{V}|\nabla u|^{2} d x+\|u\|_{\infty}^{2} \int_{V}\left|\nabla \psi_{\delta}\right|^{2} d x \\
& \leq C(|V|+\delta)
\end{aligned}
$$

Copyright $@$ by SIAM. Unauthorized reproduction of this article is prohibited. 
and $V$ and $\delta$ can be chosen arbitrarily small, by choosing diagonal sequences we see that every $u \in C^{1}(\bar{\Omega})$ and hence in fact every $u \in H^{1}(\Omega)$ can be approximated by sequences $\left(1-\psi_{\delta_{k}}\right) u_{k}, u_{k} \in X_{k}$.

6. Concluding remarks. The preceding analysis shows that, whereas the density of the LME approximation scheme in the interior of the domain follows directly from the general results for meshfree approximation schemes, the density of the scheme up to the boundary is a matter of considerable delicacy. This situation strongly suggests relaxing the positivity constraint and allowing for signed basis functions. This relaxation is also required for the formulation of higher order approximation schemes, as noted by $[2,10]$. Indeed, in the finite element limit basis functions of quadratic order and higher are signed functions in general. As an additional bonus, signed basis functions enable the consideration of general - not necessarily convex-domains. These extensions are pursued in a follow-up publication [5], where LME-type approximation schemes of arbitrary order and smoothness are derived and their convergence properties are analyzed using the general analysis framework developed in this paper.

\section{REFERENCES}

[1] R. A. Adams and J. J. F. Fournier, Sobolev Spaces, 2nd ed., Academic Press, Boston, 2003.

[2] M. Arroyo and M. Ortiz, Local maximum-entropy approximation schemes: A seamless bridge between finite elements and meshfree methods, Internat. J. Numer. Methods Engrg., 65 (2006), pp. 2167-2202.

[3] M. Arroyo and M. Ortiz, Local maximum entropy approximation schemes, in Meshfree Methods for Partial Differential Equations III, Lect. Notes Comput. Sci. and Eng. 57, Springer, Berlin, 2007.

[4] I. BABušKa, U. BANERJEe, AND J. E. Osborn, Survey of meshless and generalized finite element methods: A unified approach, Acta Numer., 12 (2003), pp. 1-125.

[5] A. Bompadre, L. E. Perotti, C. J. Cyron, and M. Ortiz, Convergent meshfree approximation schemes of arbitrary order and smoothness, Comput. Methods Appl. Mech. Engrg., 221/222 (2012), pp 80-103.

[6] P. G. Ciarlet, The Finite Element Method for Elliptic Problems, Classics Appl. Math. 40, SIAM, Philadelphia, 2002.

[7] P. G. Ciarlet and P. A. Raviart, General Lagrange and Hermite interpolation in $\mathbb{R}^{n}$ with applications to finite element methods, Arch. Ration. Mech. Anal., 46 (1972), pp. 177-199.

[8] S. Conti, P. Hauret, and M. Ortiz, Concurrent multiscale computing of deformation microstructure by relaxation and local enrichment with application to single-crystal plasticity, Multiscale Model. Simul., 6 (2007), pp. 135-157.

[9] J. A. Cottrell, A. Reali, Y. Bazilevs, and T. J. R. Hughes, Isogeometric analysis of structural vibrations, Comput. Meth. Appl. Mech. and Engrg., 195 (2006) pp. 5257-5296.

[10] C. J. Cyron, M. Arroyo, And M. Ortiz, Smooth, second order, non-negative meshfree approximants selected by maximum entropy, Internat. J. Numer. Methods Engrg., 79 (2009), pp. $1605-1632$.

[11] G. DAL MASO, Introduction to Г-Convergence, Birkhäuser Boston, Boston, MA, 1993.

[12] L. C. Evans and R. F. Gariepy, Measure Theory and Fine Properties of Functions, CRC Press, Boca Raton, FL, 1992.

[13] D. González, E. Cueto, and M. Doblaré, A higher order method based on local maximum entropy approximation, Internat. J. Numer. Methods Engrg., 83 (2010), pp. 741-764.

[14] A. Huerta, T. Belytschko, S. Fernández-Méndez, and T. Rabczuk, Meshfree methods, in Encyclopedia of Computational Mechanics, Wiley, New York, 2004, pp. 279-309.

[15] E. T. JAYNES, Information theory and statistical mechanics, Phys. Rev., 106 (1957), pp. 620630.

[16] A. I. Khinchin, Mathematical Foundations of Information Theory, Dover, New York, 1957.

[17] B. Li, F. HABbAL, AND M. ORTIZ, Optimal transportation meshfree approximation schemes for fluid and plastic flows, Internat. J. Numer. Methods Engrg., 83 (2010), pp. 1541-1579.

[18] W. K. Liu, S. Li, And T. Belytschko, Moving least square reproducing kernel methods Part I: Methodology and convergence, Comput. Methods Appl. Mech. Engrg., 143 (1997), pp. 113154 .

Copyright $@$ by SIAM. Unauthorized reproduction of this article is prohibited. 
[19] J. M. Melenk, On approximation in meshless methods, in Frontiers of Numerical Analysis, James F. Blowey and Alan W. Craig, eds., Universitext, Springer, Berlin, Heidelberg, 2005, pp. 65-141.

[20] W. Quak, D. González, E. Cueto, and A. H. van den Boogand, On the use of local max-ent shape functions for the simulation of forming processes, in Proceedings of the $\mathrm{X}$ International Conference on Computational Plasticity (COMPLAS X), 2009.

[21] V. T. RAJAn, Optimality of the Delaunay triangulation in $\mathbb{R}^{d}$, Discrete Comput. Geom., 12 (1994), pp. 189-202.

[22] R. T. Rockafellar, Convex Analysis, Princeton University Press, Princeton, NJ, 1970.

[23] W. Rudin, Functional Analysis, 2nd ed., McGraw-Hill, Hightstown, NJ, 1991.

[24] R. Schaback And H. Wendland, Kernel techniques: From machine learning to meshless methods, Acta Numer., 15 (2006), pp. 543-639.

[25] C. E. Shannon, A mathematical theory of communication, The Bell System Technical Journal, 27 (1948), pp. 379-423.

[26] G. Strang and G. Fix, An Analysis of the Finite Element Method, Prentice-Hall, Englewood Cliffs, NJ, 1973.

[27] N. SuKumar, Construction of polynomial interpolants: a maximum entropy approach, Internat. J. Numer. Methods Engrg., 61 (2004), pp. 2159-2181.

[28] N. Sukumar, Maximum entropy approximation, in Proceedings of the 25th International Workshop on Bayesian Inference and Maximum Entropy Methods in Science and Engineering, AIP Conf. Proc. 803, 2005, pp. 337-344.

[29] N. Sukumar and R. J.-B. Wets, Deriving the continuity of maximum-entropy basis functions via variational analysis, SIAM J. Optim., 18 (2007), pp. 914-925.

[30] N. Sukumar And R. Wright, Overview and construction of meshfree basis functions: From moving least squares to entropy approximants, Internat. J. Numer. Methods Engrg., 70 (2007), pp. 181-205.

Copyright (c) by SIAM. Unauthorized reproduction of this article is prohibited. 